2015

\title{
A comparison of ion beam measurements by retarding field energy analyzer and laser induced fluorescence in helicon plasma devices
}

N. Gulbrandsen

Å. Fredriksen

J. Carr

E. Scime

Follow this and additional works at: https://researchrepository.wvu.edu/faculty_publications

\section{Digital Commons Citation}

Gulbrandsen, N.; Fredriksen, Å.; Carr, J.; and Scime, E., "A comparison of ion beam measurements by retarding field energy analyzer and laser induced fluorescence in helicon plasma devices" (2015). Faculty Scholarship. 26.

https://researchrepository.wvu.edu/faculty_publications/26 


\title{
A comparison of ion beam measurements by retarding field energy analyzer and laser induced fluorescence in helicon plasma devices
}

\author{
N. Gulbrandsen, ${ }^{1, a)} \AA$ A. Fredriksen, ${ }^{1}$ J. Carr, Jr., ${ }^{2,3}$ and E. Scime ${ }^{2}$ \\ ${ }^{1}$ Department of Physics and Technology, UiT The Arctic University of Norway, 9037 Troms $\phi$, Norway \\ ${ }^{2}$ Department of Physics and Astronomy, West Virginia University, Morgantown, West Virginia 26506, USA \\ ${ }^{3}$ Department of Physics, Texas Lutheran University, Seguin, Texas 78155, USA
}

(Received 12 December 2014; accepted 20 February 2015; published online 6 March 2015)

\begin{abstract}
Both Laser-Induced Fluorescence (LIF) and Retarding Field Energy Analyzers (RFEA) have been applied to the investigation of beams formed in inductively coupled helicon plasmas. While the LIF technique provides a direct measurement of the velocity distribution in the plasma, the RFEA measures ion flux as a function of a retarding potential. In this paper, we present a method to compare the two techniques, by converting the LIF velocity distribution to an equivalent of a RFEA measurement. We applied this method to compare new LIF and RFEA measurements in two different experiments; the Hot Helicon Experiment (HELIX) - Large Experiment on Instabilities and Anisotropies (LEIA) at West Virginia University and Njord at University of Troms $\varnothing$. We find good agreement between beam energies of the two methods. In agreement with earlier observations, the RFEA is found to measure ion beams with densities too low for the LIF to resolve. In addition, we present measurements of the axial development of the ion beam in both experiments. Beam densities drop exponentially with distance from the source, both in LIF and RFEA measurements. The effective quenching cross section from LIF in LEIA is found to be $\sigma_{b, *}=4 \times 10^{-19} \mathrm{~m}^{2}$, and the effective beam collisional cross sections by RFEA in Njord to be $\sigma_{b}=1.7 \times 10^{-18} \mathrm{~m}^{2}$. C 2015 AIP Publishing LLC. [http://dx.doi.org/10.1063/1.4913990]
\end{abstract}

\section{INTRODUCTION}

Extensive measurements ${ }^{1-7}$ and analysis $^{8-11}$ of beam formation in current-free double layers (CFDLs) have been performed since the first beam observations in inductively coupled helicon plasmas. ${ }^{12-14}$ Such beams are typically observed a short distance after the source plasma has flowed into an expansion chamber downstream from the source. Control parameters usually must be set to low collisionality, and magnetic field lines normally expand from the magnetized source plasma into the low-magnetized or unmagnetized plasma in the expansion chamber.

To obtain the beam data, retarding field energy analyzers (RFEAs) are most often employed. ${ }^{12}$ Such probes measure ion current to a collector as a function of applied potential to a so-called discriminator grid in front of it, such that a representation of the ion velocity distribution can be obtained. Often, these probes can be rotated to measure the ion distribution in different directions, assuming that the probe scale is much smaller than the particle gyroradius. However, for a closer investigation of the ion distributions, the probe has the drawback that its housing is relatively large and commonly grounded and therefore surrounded by a sheath with a potential drop of about $10 \mathrm{~V}$ and up to $100 \mathrm{~V}$ in some cases. In addition, RF-produced plasmas exhibit oscillations in the plasma potential. These two effects distort and broaden the measured distribution to the extent that it is generally not possible to truthfully recover the ion velocity distribution in the undisturbed plasma. Instead, the probe

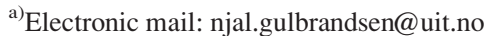

measures the ion distribution entering the probe after having passed the sheath. ${ }^{15}$

Laser Induced Fluorescence (LIF) diagnosis ${ }^{16}$ is not intrusive, unlike the rather large RFEA probes, and it provides direct measurements of the velocity distribution of metastable ions, which is interpreted as a representation of the velocity distribution of the entire ion population. ${ }^{17}$ The availability of tunable diode lasers with more than $10 \mathrm{~mW}$ effective power has made LIF-diagnostics more feasible for this type of plasmas. LIF diagnostics using tunable diode lasers were first implemented by Severn et al. ${ }^{16}$ Low-power LIF using tunable diode lasers was later implemented at West Virginia University $(\mathrm{WVU})^{18}$ and was soon afterward applied in studies of flow. ${ }^{19}$ These measurements were followed by LIF investigations of beam plasmas at Princeton Plasma Physics Laboratory (PPPL) ${ }^{14}$ and Australian National University (ANU). ${ }^{1,3}$

Still, LIF diagnosis of such beam-plasma systems is less common than RFEA measurements. Direct comparisons between the two diagnostics are rarely encountered, ${ }^{15}$ and the method of comparison has to our knowledge not yet been investigated in detail. Earlier efforts to compare the two types of diagnostics have been based on fitting an expression for an idealized collector current from two drifting Maxwellian populations to the measurements. ${ }^{15,20}$

In this article, we present for the first time a new method for detailed comparison by converting the LIF signal to a RFEA-like signal using a simple sheath model. The advantage of this method is that we start with the more accurate velocity distribution from the LIF measurements, then calculate the distribution an ideal probe would see and then compare it to real measurements by the RFEA. Thus, we 
avoid assuming an idealized sheath model to interpret the RFEA-measurements. We do not perform any fitting of parameters and thereby avoid making assumptions of background or beam distribution shape. In addition, flow is already included in the LIF-measurements. The plasma potential has to be obtained from an RFEA-measurement, or another probe measurement, and is the most uncertain parameter in this procedure. We applied this method to new LIF and RFEA measurements in two different devices in which a beam can be maintained by a CFDL, namely, the Hot Helicon Experiment - Large Experiment on Instabilities and Anisotropies (HELIX-LEIA) system at $\mathrm{WVU}^{21,22}$ and the Njord device at University of Troms $\varnothing(\mathrm{UiT}){ }^{4}$

We also present the first LIF measurements of ion beams in the Njord device, performed with a low power diode laser.

In Sec. II, we describe the experimental setups with LIF and RFEA diagnostics of the HELIX-LEIA and the Njord devices, respectively. Furthermore, we describe the analysis of the LIF and RFEA measurements along with the description of the method we developed for the comparison between the two diagnostics techniques. In Sec. III, LIF and RFEA data obtained in HELIX-LEIA and Njord are described, and axial, radial, and pressure dependence of the derived plasma parameters are presented. Section IV contains a discussion of the similarities and differences between the diagnostics methods and the behavior of the measured quantities with respect to the given parameters.

\section{EXPERIMENTAL SETUP}

The experiments were conducted in two different plasma devices, the HELIX-LEIA device at WVU and the Njord device at the UiT. The devices are similar in that they are cylindrical and their helicon sources are operated in inductive mode, producing a high-density low-temperature plasma which expands into a larger chamber. However, there are differences in dimensions as well as magnetic field. Below, a more detailed description of the two sources and their diagnostics systems are given.

\section{A. The WVU HELIX-LEIA-system}

The HELIX ${ }^{21}$ at WVU, shown in Figure 1, consists of a $61 \mathrm{~cm}$ long Pyrex tube, $10 \mathrm{~cm}$ in diameter, connected to a $91 \mathrm{~cm}$ long, $15 \mathrm{~cm}$ diameter stainless-steel chamber. This chamber expands into a $4.5 \mathrm{~m}$ long, $2 \mathrm{~m}$ diameter space simulation chamber LEIA. ${ }^{22}$ Two turbomolecular pumps at the other end of LEIA keeps the base pressure at $10^{-7}$ Torr. Argon is injected through a precision MKS mass flow controller into the stainless-steel chamber of HELIX (downstream of the source region). Flow rates in the range of $1.7-3.0 \mathrm{sccm}$ were used in this study, resulting in a pressure range of 0.2-0.9 mTorr in HELIX and 0.07-0.09 mTorr in LEIA. An RF amplifier supplies $650 \mathrm{~W}$ of RF-power at $9.5 \mathrm{MHz}$ to the plasma through a $\pi$ matching circuit. A $19 \mathrm{~cm}$ half wave helical antenna couples the RF-energy into the plasma (Figure 1).

At HELIX, ten water-cooled electromagnets produce a steady state axial magnetic field of $0-1300 \mathrm{G}$. A magnetic field of $900 \mathrm{G}$ was used in the measurements reported here. LEIA has seven water-cooled electromagnets, but these were

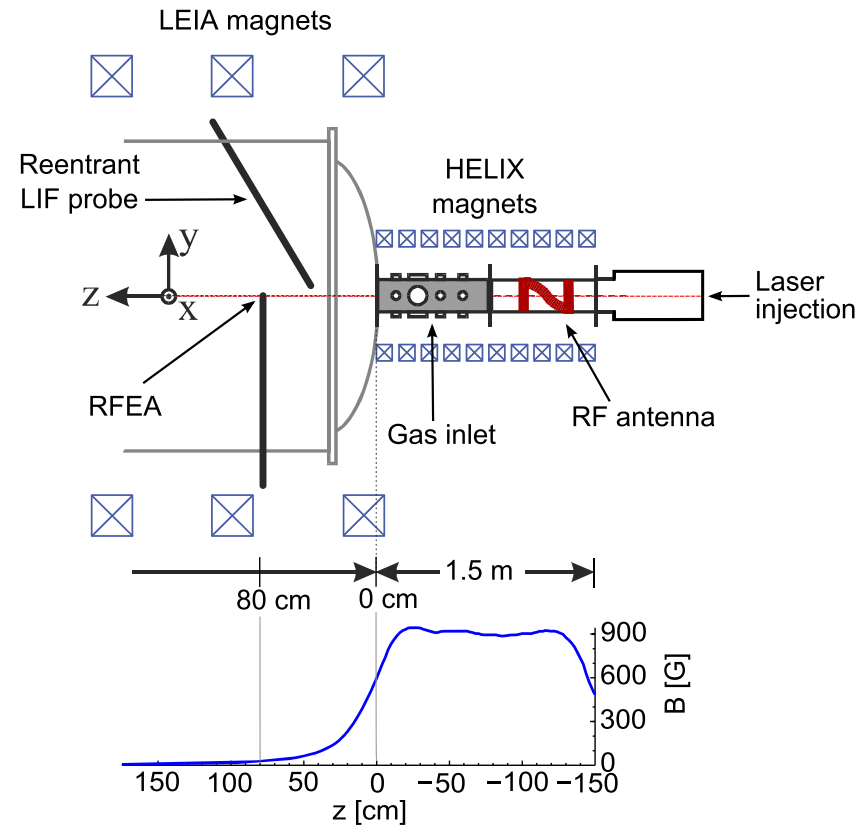

FIG. 1. The WVU HELIX-LEIA-system. Origin of the $\mathrm{z}$-axis is placed at the junction between the HELIX and the LEIA systems. Below, a plot of the axial magnetic field is shown.

not used in this study. Hence, the magnetic field is given by the HELIX magnets only. The junction between HELIX and LEIA is defined as $z=0$ and positive $z$ is in the direction of gas flow from the HELIX-source into LEIA. At $z=80 \mathrm{~cm}$, the magnetic field is about $20 \mathrm{G}$. The main plasma parameters are given in Table I.

\section{B. UiT Njord system}

The Njord device (Figure 2) at UiT is an inductively coupled helicon plasma device. ${ }^{4,23}$ The source consists of a $30 \mathrm{~cm}$ long, $13 \mathrm{~cm}$ diameter Pyrex tube with a saddle antenna wrapped around it, coupling $1000 \mathrm{~W}$ of RF-power at $13.56 \mathrm{MHz}$ to the argon gas. Around the source tube, two magnetic field coils with currents of 5 and $6 \mathrm{~A}$ produce a maximum magnetic field of $200 \mathrm{G}$ in the source. The plasma expands through a $20 \mathrm{~cm}$ diameter and $8 \mathrm{~cm}$ long port into a $120 \mathrm{~cm}$ long, $60 \mathrm{~cm}$ diameter expansion chamber. A turbomolucular pump connected to the expansion chamber keeps the base pressure at $10^{-6}$ Torr.

The outer edge of the first source coil defines $z=0$, and positive $z$ is in the direction of the gas flow, from source to the expansion chamber. The source itself starts at $z=4 \mathrm{~cm}$.

A field coil with a current of 5 A placed at $z=60 \mathrm{~cm}$ provides a downstream magnetic field of about $30 \mathrm{G}$. This field is to prevent electrons from the near wall region of the source from getting lost at the walls of the large port. Argon gas was fed to the source through an inlet in a grounded aluminum end plate of the source (at $z=4 \mathrm{~cm}$ in Figure 2). The flow was controlled by an Omega mass flow controller and kept between 1.1 and $2.0 \mathrm{sccm}$ in this study, giving rise to pressures of 0.2-0.4 mTorr in the expansion chamber.

An axial probe feedthrough, indicated in Figure 2, is fitted with an RFEA probe with the aperture facing the source. The RFEA can be moved axially between $z=36 \mathrm{~cm}$ and 
TABLE I. Comparing typical parameters.

\begin{tabular}{|c|c|c|c|c|}
\hline & HELIX (source) & LEIA at $80 \mathrm{~cm}$ & Njord (source) & Njord (downstream) \\
\hline Pressure, $P$ & $7.1 \times 10^{-4}$ Torr & $9.9 \times 10^{-5}$ Torr & & $3.1 \times 10^{-4}$ Torr \\
\hline Magnetic field, $B$ & $900 \mathrm{G}$ & $22 \mathrm{G}$ & $200 \mathrm{G}$ & $29 \mathrm{G}$ \\
\hline RF power & $650 \mathrm{~W}$ & & $1000 \mathrm{~W}$ & \\
\hline RF frequency & $9.5 \mathrm{MHz}$ & & $13.56 \mathrm{MHz}$ & \\
\hline Plasma density, $n_{i}$ & $3 \times 10^{11} \mathrm{~cm}^{-3}$ & $7 \times 10^{9} \mathrm{~cm}^{-3}$ & $2 \times 10^{11} \mathrm{~cm}^{-3}$ & $3 \times 10^{10} \mathrm{~cm}^{-3}$ \\
\hline Electron temperature, $T_{e}$ & $3 \mathrm{eV}$ & $4 \mathrm{eV}$ & $8 \mathrm{eV}$ & $6 \mathrm{eV}$ \\
\hline Ion temperature, $T_{i}$ & $0.7 \mathrm{eV}$ & $1 \mathrm{eV}$ & & $0.2 \mathrm{eV}$ \\
\hline Floating potential, $V_{f}$ & $2.0 \mathrm{~V}$ & $-1.5 \mathrm{~V}$ & $27 \mathrm{~V}$ & $10 \mathrm{~V}$ \\
\hline Plasma potential, $V_{p}$ & $40 \mathrm{~V}$ & $14 \mathrm{~V}$ & $60 \mathrm{~V}$ & $45 \mathrm{~V}$ \\
\hline Debye length, $\lambda_{D}$ & $24 \mu \mathrm{m}$ & $170 \mu \mathrm{m}$ & & $110 \mu \mathrm{m}$ \\
\hline Electron cyclotron frequency, $\omega_{c e}$ & $1.6 \times 10^{10} \mathrm{rad} / \mathrm{s}$ & $3.9 \times 10^{8} \mathrm{rad} / \mathrm{s}$ & & $5.1 \times 10^{8} \mathrm{rad} / \mathrm{s}$ \\
\hline Ion cyclotron frequency, $\omega_{c i}$ & $2.2 \times 10^{5} \mathrm{rad} / \mathrm{s}$ & $5.3 \times 10^{3} \mathrm{rad} / \mathrm{s}$ & & $7.0 \times 10^{3} \mathrm{rad} / \mathrm{s}$ \\
\hline Electron thermal velocity, $v_{t h, e}$ & $1.1 \times 10^{6} \mathrm{~m} / \mathrm{s}$ & $1.2 \times 10^{6} \mathrm{~m} / \mathrm{s}$ & & $1.5 \times 10^{6} \mathrm{~m} / \mathrm{s}$ \\
\hline Ion thermal velocity, $v_{t h, i}$ & $1800 \mathrm{~m} / \mathrm{s}$ & $2300 \mathrm{~m} / \mathrm{s}$ & & $1000 \mathrm{~m} / \mathrm{s}$ \\
\hline Electron Larmor radius, $r_{L, e}$ & $6.7 \times 10^{-5} \mathrm{~m}$ & $3.0 \times 10^{-3} \mathrm{~m}$ & & $2.9 \times 10^{-3} \mathrm{~m}$ \\
\hline Ion Larmor radius, $r_{L, i}$ & $8.5 \times 10^{-3} \mathrm{~m}$ & $0.43 \mathrm{~m}$ & & $0.14 \mathrm{~m}$ \\
\hline Bohm velocity, $u_{B}$ & $2780 \mathrm{~m} / \mathrm{s}$ & $3030 \mathrm{~m} / \mathrm{s}$ & $4450 \mathrm{~m} / \mathrm{s}$ & $3870 \mathrm{~m} / \mathrm{s}$ \\
\hline Plasma frequency, $\omega_{p e}$ & $3.2 \times 10^{10} \mathrm{rad} / \mathrm{s}$ & $4.7 \times 10^{9} \mathrm{rad} / \mathrm{s}$ & $2.5 \times 10^{10} \mathrm{rad} / \mathrm{s}$ & $9.8 \times 10^{9} \mathrm{rad} / \mathrm{s}$ \\
\hline Plasma parameter, $N_{D}$ & $1.7 \times 10^{4}$ & $1.5 \times 10^{5}$ & $9.3 \times 10^{4}$ & $1.5 \times 10^{5}$ \\
\hline Plasma $\beta$ & $1.1 \times 10^{-5}$ & $6.3 \times 10^{-4}$ & & $3.0 \times 10^{-4}$ \\
\hline Beam mean free path $\lambda_{b}{ }^{\mathrm{a}}$ & $7.3 \mathrm{~cm}$ & $53 \mathrm{~cm}$ & & $16 \mathrm{~cm}$ \\
\hline
\end{tabular}

${ }^{\mathrm{a}}$ For $25 \mathrm{eV}$ beam. ${ }^{24}$

$z=56 \mathrm{~cm}$. Typical parameters for the Njord device are given in Table I.

\section{RFEA}

Three different RFEAs were used to measure Ion Distribution Functions (IDF) in this study. In measurements

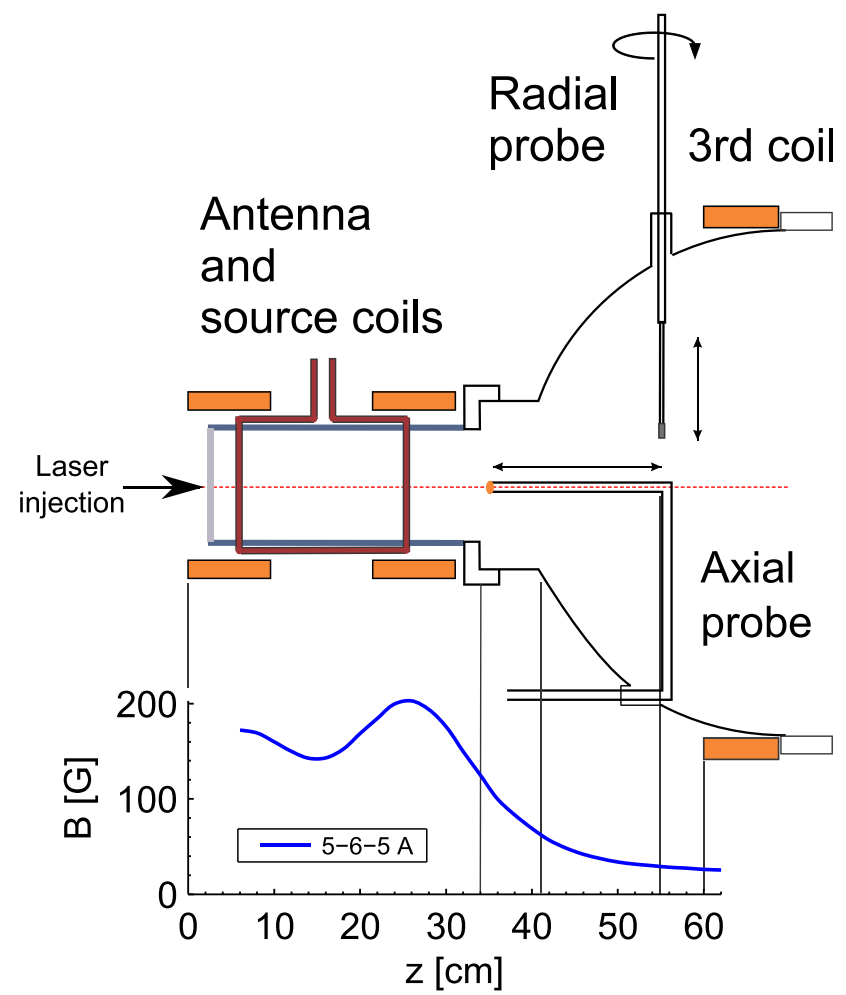

FIG. 2. The UiT Njord device. Origin of the z-axis is placed at the edge of the first source coil. Below, a plot of the axial magnetic field used in the experiment. at WVU, we used an RFEA with a ceramic housing. At UiT, we used two different RFEAs, both with a grounded brass housing, of which one was mounted at a radial port looking perpendicular to the probe shaft and one was looking opposite to the probe shaft and mounted on the axial feedthrough.

\section{Ceramic RFEA}

For the WVU measurements, we used a RFEA with a ceramic housing $(35 \times 22 \times 15 \mathrm{~mm})$ connected to a grounded probe shaft. The probe was oriented perpendicular to the shaft and could be rotated $360^{\circ}$ around the axis to look in different directions. The probe was mounted at a radial port at $z=80 \mathrm{~cm}$ in LEIA (Figure 1). This RFEA had four grids of stainless steel mesh with a transmission factor of $44 \%$ and $50 \mu \mathrm{m}$ wire spacing. Each grid was spotwelded to a $0.4 \mathrm{~mm}$ thick brass spacer. This probe had two front grids $0.4 \mathrm{~mm}$ apart, both electrically grounded. Two configurations of this probe were used, one with a $5 \mathrm{~mm}$ diameter aperture and one with a $7 \mathrm{~mm}$ diameter aperture. The remaining brass spacers had an inner diameter of $7 \mathrm{~mm}$. Between the other grids, $0.5 \mathrm{~mm}$ thick ceramic spacers were used so that the distance between the grids was $0.9 \mathrm{~mm}$. The ceramic spacer in front of the collector plate had an opening diameter to match the front aperture, either $5 \mathrm{~mm}$ or $7 \mathrm{~mm}$. This was done to maximize the beam signal relative to the background. The repeller grid was biased to $-100 \mathrm{~V}$ using a power supply, while the discriminator was swept from $-80 \mathrm{~V}$ to $80 \mathrm{~V}$ in 1000 steps. The collector was kept at $-9 \mathrm{~V}$ using a battery. At each step, the collector current was measured across a $100 \mathrm{k} \Omega$ resistor and digitized with a 16-bit DAQ from National Instruments. The grid configuration of the ceramic RFEA is illustrated in Figure 3(a). 


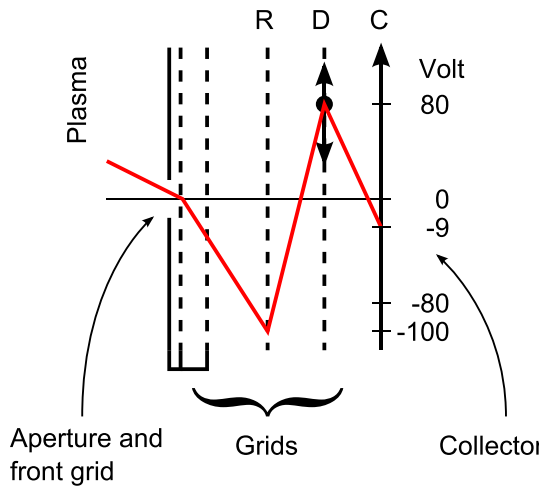

(a)

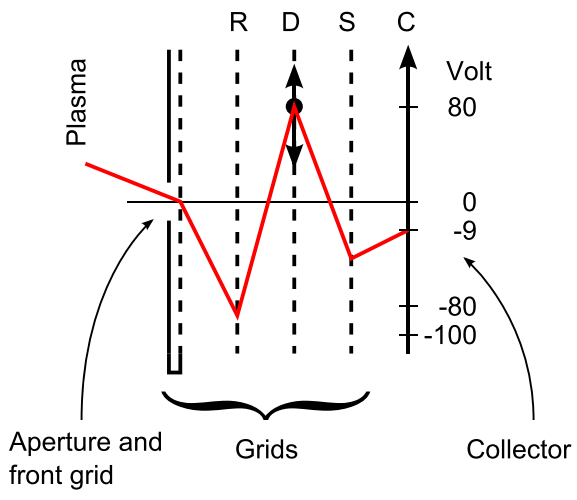

(b)

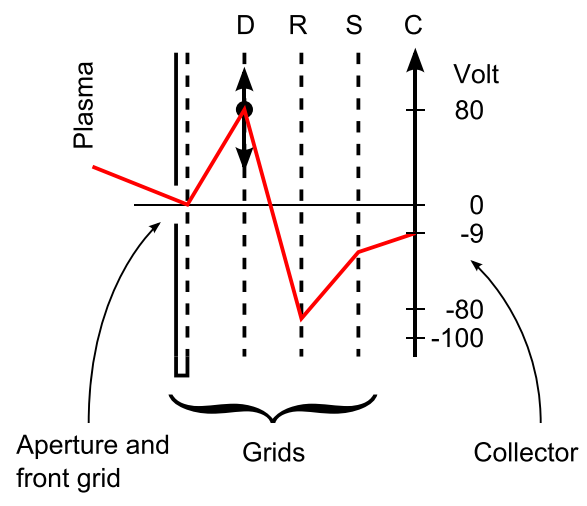

(c)

FIG. 3. Illustrations of the grid configurations in the different RFEA-probes. R is the repeller grid, D the discriminator grid, $\mathrm{S}$ the secondary electron repeller, and $\mathrm{C}$ is the collector.

\section{Brass RFEA}

For the radial measurements at UiT, we used an RFEA with a grounded brass housing with a cylindrical shape, diameter of $16 \mathrm{~mm}$, and thickness of $10 \mathrm{~mm}$. The probe has four grids, three of stainless steel mesh with a transmission factor of $44 \%$, and the fourth, the repeller grid, was made of a nickel mesh with transparancy of $67 \%$. The meshes are spotwelded to $0.3 \mathrm{~mm}$ thick brass spacers with $0.3 \mathrm{~mm}$ ceramic spacers in between, resulting in a distance of $0.6 \mathrm{~mm}$ between the grids. The front grid was electrically connected to the housing and thereby grounded. The front aperture was $2 \mathrm{~mm}$. The ceramic spacer in front of the collector limited the collector area to $3 \mathrm{~mm}$ diameter. The repeller grid was biased to $-86 \mathrm{~V}$ using a power supply, while the discriminator was swept from $-10 \mathrm{~V}$ to $120 \mathrm{~V}$ in 1000 steps using an amplifier and a series of batteries. A secondary repeller biased to $-20 \mathrm{~V}$ using a power supply was used to keep secondary electrons from leaving the collector. The collector was kept at $-9 \mathrm{~V}$ using a battery. At each step, the collector current was measured across a $100 \mathrm{k} \Omega$ resistor and digitized with a 16-bit DAQ from National Instruments. The grid configuration of the brass RFEA is illustrated in Figure 3(b).

\section{Axial RFEA}

For the axial measurements at UiT, we used a larger RFEA (Figure 3(c)) with a grounded brass housing mounted so it always faced the source. The housing was cylindrical with a diameter of $13 \mathrm{~mm}$ and length of $25 \mathrm{~mm}$. This RFEA had four grids of stainless steel with a transmission factor of $44 \%$, each spotwelded to a $0.5 \mathrm{~mm}$ thick brass spacer. The front aperture opening was $1 \mathrm{~mm}$. Between each grid, there was a $0.25 \mathrm{~mm}$ thick spacer so that the total distance between the grids becomes $0.75 \mathrm{~mm}$. The spacer had an inner diameter of $10 \mathrm{~mm}$ which limited the collector area. When using the same grid configuration as the radial brass RFEA, this probe produced a large amount of noise in the current signals. We therefore chose to use this probe with the discriminator grid in front of the repeller grid (Figure 3(c)). This configuration produced slightly sharper distributions and less noise. This type of configuration with discriminator in front of the repeller has earlier been used successfully by Gahan et $a l^{25}$ Except for these differences, this probe was used with exactly the same parameters as the radial brass RFEA; the repeller biased to $-86 \mathrm{~V}$, discriminator was swept from $-10 \mathrm{~V}$ to $120 \mathrm{~V}$, and the collector was kept at $-9 \mathrm{~V}$. At each step, the collector current was measured across a $100 \mathrm{k} \Omega$ resistor and digitized with a 16-bit DAQ from National Instruments.

\section{Analyzing RFEA measurements}

An RFEA for ions measures a collector current that is proportional to the ion flux to the collector, which is assumed to be proportional to the ion flux at the probe entrance with a cutoff for velocities that can not overcome the discriminator voltage.

If we assume a planar (one dimensional) plasma sheath, the current to the collector in an RFEA is given as ${ }^{26-28}$

$$
I\left(v_{s, \min }\right)=A e \int_{v_{s, \min }}^{\infty} v_{s} f_{s}\left(v_{s}\right) d v_{s},
$$

where $A$ is a constant depending on the front-plate aperture and the analyzer's transmission factor. $v_{s}$ and $f_{s}\left(v_{s}\right)$ are the ion velocity and the ion velocity distribution function at the probe entrance, respectively, behind the plasma sheath, and not in the plasma itself. The minimum velocity relates to the discriminator voltage, $V_{d}$, as $v_{s, \min }=\sqrt{2 e V_{d} / m_{i}}$, where $m_{i}$ is the ion mass and $e$ the elementary charge. From this, we see that the ion velocity distribution function at the probe entrance is proportional to the derivative of the collector current with respect to $V_{d}{ }^{28}$

$$
f_{s}\left(v_{s, \min }\right)=-\frac{m_{i}}{A e^{2}} \frac{\mathrm{d} I\left(V_{d}\right)}{\mathrm{d} V_{d}} \quad \text { for } \quad v_{s, \min }>0 .
$$

To find the ion velocity distribution in the plasma in front of the sheath, we have to make some assumptions about the sheath. The simplest approach is to model a plasma sheath as a simple planar electrical potential drop where the potential in the plasma stays at the plasma potential, $V_{p}$, and the probe surface is grounded. This will add a kinetic energy of $e V_{p}$ to 
the ions so that the velocity at the probe surface, $v_{s}$, is given as

$$
v_{s}=\sqrt{v^{2}+\frac{2 e V_{p}}{m_{i}}}
$$

where $v$ represents the velocity in the plasma before the potential drop of the sheath. This expression is, of course, valid for $v>0$ only, since ions moving away from the probe never will reach the surface in this model. This simplified model is for positive values of $v$ consistent with the analytical solution to the more sophisticated Emmert's sheath model ${ }^{29}$ which also takes the preesheath into account. By assuming no collisions and flux conservation through the sheath, one finds that ${ }^{29}$

$$
f_{s}\left(v_{s}\right)=f(v) \text { for } \quad v>0,
$$

where $f(v)$ is the ion velocity distribution in the plasma. Theoretically, it should be possible to derive the ion velocity distribution in a plasma from RFEA-measurements, but in practice, the inversion of Eq. (3) makes the velocity very sensitive to the effective plasma potential in front of the probe, $V_{p}$. Therefore, RFEA-measurements are usually presented as plots of $f_{s}\left(V_{d}\right)$ (or rather differential ion flux, $\left.-\mathrm{d} I / \mathrm{d} V_{d}\right)$ plotted as a function of discriminator voltage, $V_{d}$. This distribution is often called the ion energy distribution function (IEDF), but this can be misleading. ${ }^{13}$ We choose to call this distribution the ion distribution function (IDF). ${ }^{30}$ This distribution is sometimes also plotted against units of energy. ${ }^{28}$ This is then the linear kinetic energy of the ions perpendicular to the discriminator grid, $E=e V_{d}=\frac{1}{2} m_{i} v_{s, \min }^{2}$, and is not necessarily the same as the total kinetic energy. The RFEA can only measure one direction of the ion velocity. For a beam, with fairly unidirectional velocity, the measurement is a good approximation of the kinetic energy, but for the slow background plasma, the ions will have significant velocity components in other directions. Earlier studies with simulations ${ }^{30,31}$ have shown that the width of the background ion distribution measured with a RFEA is highly sensitive to the effective acceptance angle of the RFEA.

Still, a grounded RFEA can be seen as measuring a crude approximation of the sum of kinetic and potential energy with some broadening of the distribution of background plasma.

Figure 4 shows a typical RFEA-measurement of a plasma with beam in the Njord device at the University of Troms $\varnothing$. The background distribution has a peak at $V_{p}=50 \mathrm{~V}$, providing an estimate of the local plasma potential, and the beam has a peak at $V_{b}=76 \mathrm{~V}$. Using $E_{b}=e\left(V_{b}-V_{p}\right)$, that should give a beam energy of $E_{b}=26 \mathrm{eV}$. The red dashed line marks the border between beam and background distribution. This border is taken as the "knee" in the slope of the collector current, identified as the point where the negative of the double derivative of the collector current has been reduced to less than $10 \%$ of its maximum. This value is chosen because there is not always a distinct minimum between the beam and the background distribution.

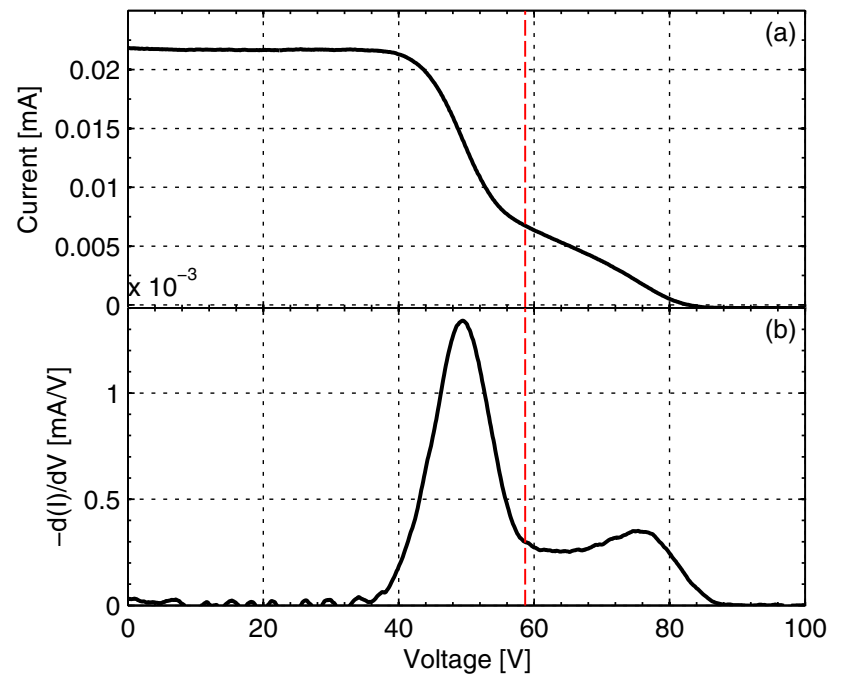

FIG. 4. RFEA-measurements in Njord at $P=0.28$ mTorr with a magnetic field of $200 \mathrm{G}$ in the source and $29 \mathrm{G}$ in the expansion chamber. RF power is $1000 \mathrm{~W}$. The measurement is taken at $z=55 \mathrm{~cm}, 11 \mathrm{~cm}$ from the end of the source and radially centered (Figure 2). The red dashed line marks the separation between beam and background distribution found by the algorithm.

\section{LIF}

LIF is a technique that utilizes the Doppler broadening of the ion absorption line of metastable ions to measure the ion velocity distribution function (IVDF). ${ }^{16,32} \mathrm{~A}$ single mode tunable laser is used to pump metastable ions which then decay by photon emission. The laser is scanned through a range of wavelengths while the intensity of the fluorescence line is measured. The frequency is converted to velocity using the simplified expression

$$
v=\lambda_{0} \Delta \nu
$$

where $v$ is the ion velocity, $\lambda_{0}$ is the excitation wavelength of the metastable at rest, and $\Delta \nu$ is the frequency difference between the excitation frequency at rest and the actual excitation frequency.

\section{WVU HELIX-LEIA LIF system}

The HELIX-LEIA LIF system consists of a $10 \mathrm{~W}$ Spectra-Physics Millennium Pro diode laser pumping a Sirah Matisse-DR tunable ring dye laser running rhodamin-6G dye. ${ }^{33}$ Typical output power is around $800 \mathrm{~mW}$. The laser is tuned to $611.6616 \mathrm{~nm}$ (vacuum wavelength) to pump the $\mathrm{Ar}$ II $3 d^{2} G_{9 / 2}$ metastable state to the $4 p^{2} F_{7 / 2}$, which then decays to the $4 s^{2} D_{5 / 2}$ state by emitting $461.086 \mathrm{~nm}$ photons. About $10 \%$ of the laser beam is split off by a beam splitter to a Bristol Instruments 621-VIS wavelength meter, while the rest is modulated with a $1 \mathrm{kHz}$ mechanical chopper and injected into the HELIX source through a fiber.

The emission is collected with a movable reentrant glass probe in the expansion chamber (Figure 1). ${ }^{34}$ The probe is kept at approximately $2 \mathrm{~cm}$ radial distance from the laser beam. The light from the probe is fed through an optical fiber to a filtered ( $1 \mathrm{~nm}$ bandwidth at $461 \mathrm{~nm})$ Hamamatsu photomultiplier tube. The signal is then fed to a Stanford Research SR830 lock-in amplifier using a time constant of $10 \mathrm{~s}$ to 
remove the background emissions. The laser was stepped through 128 different wavelengths in a total range of $20-30 \mathrm{GHz}(12-15 \mathrm{~km} / \mathrm{s})$, and the signal from the lock-in amplifier was sampled after it had stabilized for $10 \mathrm{~s}$. The filtered signal was then recorded with a computer together with the wavelength from the wavelength meter.

\section{UiT LIF-system}

The UiT LIF system consists of a Toptica DL Pro tunable single-mode-diode laser with a nominal wavelength of $668 \mathrm{~nm}$ and typical output power of $22 \mathrm{~mW}$. Ten percent of the beam is split off by a beam splitter into a Bristol Instruments 621-VIS wavelength meter, while the remaining light is modulated by a $1 \mathrm{kHz}$ mechanical chopper and then fed via two mirrors into the plasma source along the $z$-axis, through a glass window. The laser is tuned to $668.6138 \mathrm{~nm}$ to pump the argon ions in the $3 d^{4} F_{7 / 2}$ metastable state to the $4 s^{4} P_{2 / 3}$ state which then decays to the $4 s^{4} P_{3 / 2}$ state by emitting $442.7244 \mathrm{~nm}$ photons. ${ }^{1}$

The collection optics is located at a window port at $z=55 \mathrm{~cm}$ (Figure 2), pointing perpendicular to the laser beam and focused at the center of the chamber. An optical fiber brings the light from the collection optics to a Hamamatsu photo multiplier with a $442 \mathrm{~nm}, 1 \mathrm{~nm}$ bandwidth interference filter. The signal from the photomultiplier is then fed into a EG\&G Instruments 7265 lock-in amplifier using a time constant of $20 \mathrm{~s}$ for the filters. The laser was stepped through 61 different wavelengths. At each step, the wavelength was kept for $100 \mathrm{~s}$ to let the signal from the lockin amplifier stabilize. It was then sampled by a 16-bit DAQ from National Instruments while the corresponding wavelength was recorded by the wavelength meter.

\section{E. Comparison between LIF with RFEA measurements}

One would think that comparing RFEA-measurements with LIF measurements should be straightforward. After all, they both measure the ion velocity distribution; the RFEA from an integrated flux as a function of discriminator voltage, and the LIF from a density of metastable as a function of Doppler shifted wavelengths/frequencies. However, there are a couple of obstacles.

First, the RFEA and LIF do not measure the same velocity distribution. LIF is measured in the unperturbed plasma, while the RFEA measures the velocity distribution at the entrance of the probe, behind the probe sheath. Second, the LIF signal is measured as a function of Doppler shifted frequencies that can easily be converted to velocities. The RFEA signal is measured as a function of discriminator voltage inside the probe, which is proportional to the square of the velocity at the probe surface. The major issue in comparing these two types of measurements are the effects of the sheath around the probe.

To successfully compare the measurements, we need to model the effect of the sheath. A realistic sheath model is complicated and would only have numerical solutions. ${ }^{35}$ There also exist some simplified models assuming one dimensional sheath and preesheath. ${ }^{29,31}$

However, an even simpler approach is to assume that the sheath represents a potential drop from the plasma (at the plasma potential) to the probe surface (at ground), and that the density in the sheath is sufficiently low so that no collisions occur in the sheath. The system then is considered to be one dimensional, so that all ion velocities are directed either away from the probe or toward the probe, and always perpendicular to the sheath. Then, the velocity at the probe surface can be found by applying energy conservation for each ion through the sheath.

Figure 5 shows an average of six consecutive LIF measurements taken at the same parameters as the RFEA measurement in Figure 4. The error-bar represents the standard deviation at each velocity step. The large peak centered around $0.2 \mathrm{~km} / \mathrm{s}$ is the background distribution. The fact that it is not centered around zero indicates that there is a small flow along the laser beam in the background plasma. The smaller population around $11 \mathrm{~km} / \mathrm{s}$ is the beam. This corresponds to a beam energy of about $23 \mathrm{eV}$.

By using the model of Eq. (3), we calculate how a given velocity distribution would look at an idealized RFEAprobe. From RFEA-measurements, we find a plasma potential of $50 \mathrm{~V}$ (Figure 4). We use this plasma potential as input to our model, so that every ion reaching the probe would be accelerated through a sheath adding a kinetic energy of $50 \mathrm{eV}$. The discriminator voltage needed to stop an ion would then be given as

$$
V_{d}=\frac{m_{i} v^{2}}{2 e}+V_{p} \quad \text { for } \quad v>0 .
$$

No ions starting with a negative velocity will ever reach the probe in this model. Hence, negative velocities are disregarded in the calculations. The modeled collector current is found by performing the integration of Eq. (1) numerically. The LIF data were used as input for the ion velocity distribution, $f(v)$, transforming them to $f_{s}\left(v_{s}\right)$ using Eq. (3).

Figure 6(a) shows the result of this integration. For $V<V_{p}$, the current is constant, reflecting the fact that the negative part of the velocity distribution is removed.

Figure 6(b) shows the negative of the derivative of the modeled collector current. This is the distribution usually derived from RFEA-measurements [Eq. (2)], which provides the ion velocity distribution as a function of discriminator voltage.

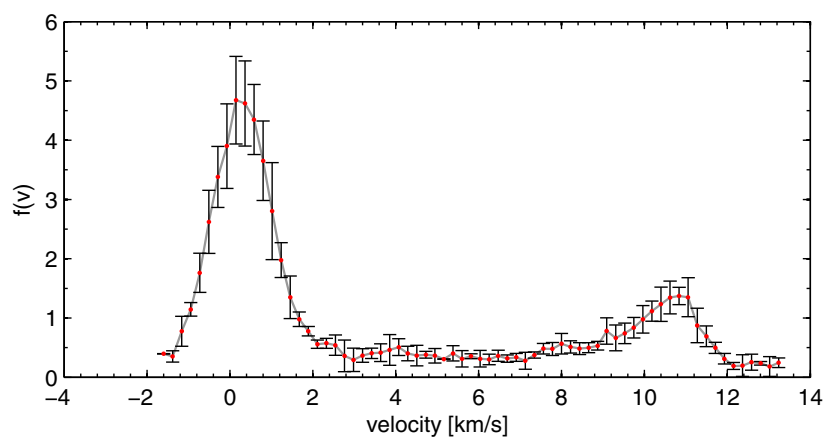

FIG. 5. LIF measurements of velocity distribution function in Njord, $f(v)$ as a function of velocity, averaged over 3-6 measurements for each data point. $P=0.28$ mTorr with a magnetic field of $200 \mathrm{G}$ in the source and $29 \mathrm{G}$ in the expansion chamber. The measurement is taken at the position $z=55 \mathrm{~cm}$, (Figure 2). 


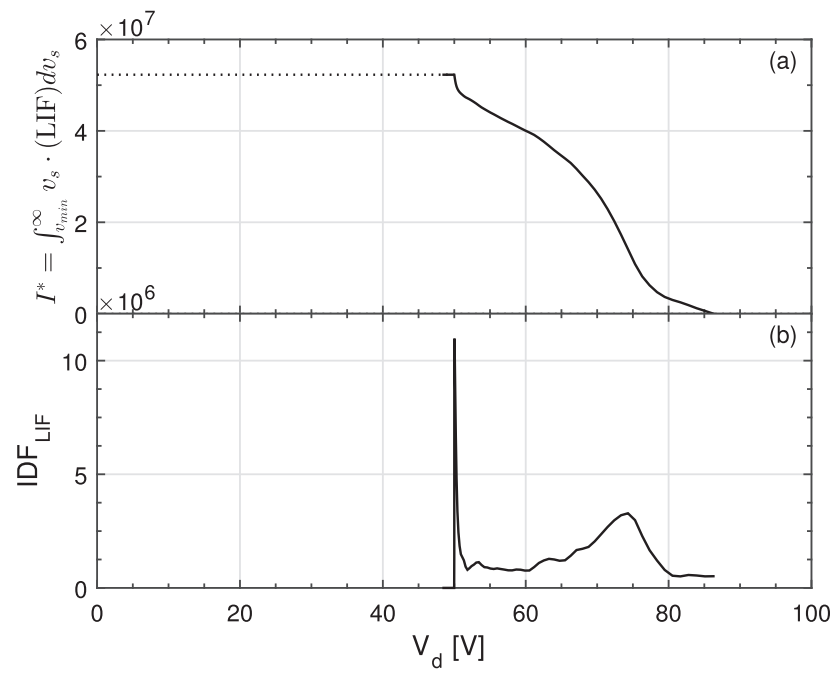

FIG. 6. (a) Model collector current calculated from the LIF measurement in Figure 5 , assuming a plasma potential, $V_{p}=50 \mathrm{~V}$, plotted with respect to discriminator potential. The dotted line is an assumed continuation to the ion saturation current. (b) The negative of the derivative of the model collector current in (a) which is proportional to the velocity distribution function plotted versus discriminator voltage.

If we compare the RFEA measurements (Figure 4) with the transformed LIF measurements (Figure 6), the most striking difference is the width of the main peak, the background distribution. While the LIF measurements give a narrow and sharp peak corresponding to an ion temperature $T_{i}=0.2 \mathrm{eV}$, the main peak of the RFEA measurements is much wider and would correspond to a temperature of around $10 \mathrm{eV}$ if it was considered real.

The beam parts of the measurements are more similar. Although the RFEA does not manage to fully resolve the beam, it is fairly close in shape to the transformed LIF data. The LIF measurement gives a beam energy of $23 \mathrm{eV}$, which is quite consistent with the beam energy from the RFEA of $E_{b}=26 \mathrm{eV}$.

There are two main reasons why a RFEA resolves the beam better than the background. First, since $V \sim v^{2}$, measuring voltage compresses the $x$-axis at low velocities relative to high velocities in the distribution. Since the probe has a fixed energy resolution, low velocities are proportionally less resolved than high velocities. Second, the beam is close to unidirectional and already has a high velocity toward the probe. It is therefore less affected by the sheath. The background distribution has velocities in all directions, and the probe has a relatively large acceptance angle $\left(\sim 50^{\circ}\right)$. Combined with a large sheath, this results in a broadening of the measured background distribution from ion focusing in the probe, ${ }^{36,37}$ and weak ion-ion collisions in the sheath. ${ }^{38}$ The broadening effect of the sheath has been investigated by particle-in-cell (PIC)-simulations. ${ }^{30,31}$ In addition, there is a slight broadening of both the background and the beam distribution by the RF-oscillations in the plasma. ${ }^{39}$

\section{MEASUREMENTS}

Comparative measurements with LIF and RFEA were carried out in LEIA with LIF at a set of axial positions from $z=36 \mathrm{~cm}$ to $z=79 \mathrm{~cm}$, while a port at $z=80 \mathrm{~cm}$ was available for RFEA measurements. Hence, axial development of the beam was obtained by means of the LIF system, and radial measurements were obtained with the RFEA. Only one position, namely, $z=79-80 \mathrm{~cm}$, was in common.

In Njord, the LIF measurements were available at only one axial position, $z=55 \mathrm{~cm}$, while RFEA measurements could be obtained both axially between $z=35$ and $56 \mathrm{~cm}$ and radially outward to $r=18 \mathrm{~cm}$.

In this section, we first report the measurements obtained in LEIA, with respect to axial and radial position, and as a function of pressure. Second, the corresponding measurements in Njord are described.

\section{A. WVU HELIX-LEIA}

\section{Axial development}

Figure 7(a) shows a series of LIF measurements taken at different positions along the central axis (z-axis) in the HELIX-LEIA device. The LIF signal is given in uncalibrated voltages from the photomultiplier, filtered through the lockin amplifier. The plasma source was supplied with a flow of $3 \mathrm{sccm}$ argon resulting in a pressure of $7.1 \times 10^{-4}$ Torr in the source (HELIX) and $9.9 \times 10^{-5}$ Torr in the expansion chamber (LEIA).
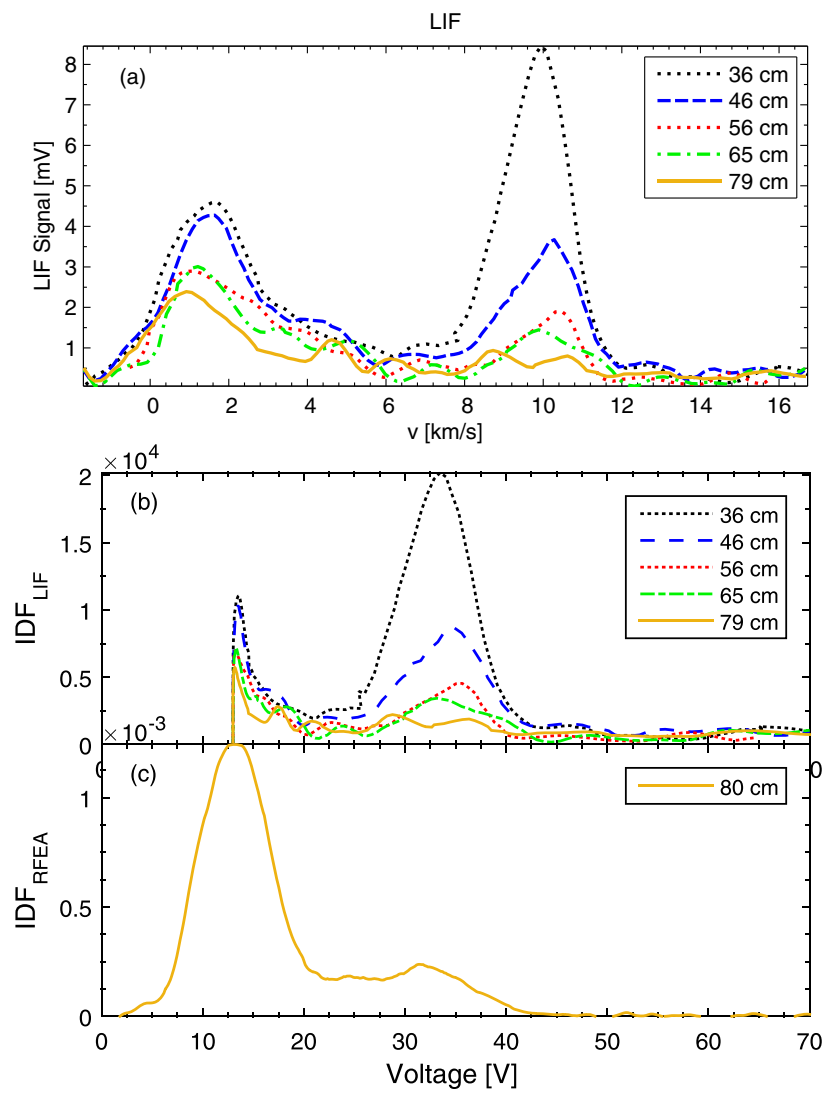

FIG. 7. (a) LIF measurements of the velocity distribution function, $f(v)$ converted from frequency to velocity, in the center of LEIA, at different distances from HELIX-LEIA junction $(z=0 \mathrm{~cm})$. The data have been smoothed with a 3rd degree, 11 point Savitzky-Golay filter to remove noise. (b) By assuming a sheath voltage of $13 \mathrm{~V}$, the ion velocity distribution converted to the equivalent of an ideal RFEA, using $V=m_{i} v^{2} / 2 e+V_{p}$. (c) Derivative of collector current from RFEA measurement at $z=80 \mathrm{~cm}$, in the center of the chamber, $r=0$. An aperture opening of $7 \mathrm{~mm}$ was used. 
The distribution with maximum seen around $1-2 \mathrm{~km} / \mathrm{s}$ is the background distribution of the downstream plasma with a small flow, while the one with a peak around $10 \mathrm{~km} / \mathrm{s}$ is the beam. The beam intensity decreases systematically with distance from the source while the beam velocity is close to constant. Only a slight shift to higher velocity for the peak of the first three scans $(z=36 \mathrm{~cm}-56 \mathrm{~cm})$ is observed, from $10.0 \mathrm{~km} / \mathrm{s}$ to $10.8 \mathrm{~km} / \mathrm{s}$. In the two last scans $(z=65 \mathrm{~cm}$ and $79 \mathrm{~cm}$ ), the beam is barely visible above the noise level.

The maximum of the background distribution also decreases by distance to the source but not so systematically. At $z=36 \mathrm{~cm}$ and $46 \mathrm{~cm}$, the maximum stays at the same level of about $4.5 \mathrm{mV}$. At $z=56 \mathrm{~cm}$, it drops down to $3 \mathrm{mV}$ and stays there until $z=65 \mathrm{~cm}$. At $z=79 \mathrm{~cm}$, the level is decreased to $2.5 \mathrm{mV}$.

Figure 7(b) shows the LIF signal in (a) converted to RFEA equivalent voltages applying the same model as for Figure 6 [Eqs. (1) and (6)] using $13 \mathrm{~V}$ as plasma potential. Figure 7(c) shows the RFEA measurements at $z=80 \mathrm{~cm}$ taken at the same time as the LIF measurement at $z=79 \mathrm{~cm}$. The background distribution has maximum at $V_{p}=13 \mathrm{~V}$, representing the local plasma potential, and the beam has a maximum at $V_{b}=32 \mathrm{~V}$, giving a beam energy of $E_{b}=19 \mathrm{eV}$. For comparison, the beam velocity from the LIF measurements of $10 \mathrm{~km} / \mathrm{s}$ equals a beam energy of $20 \mathrm{eV}$.

The major difference between the LIF and the RFEA in Figures 7(a) and 7(b) is the much wider background distribution of the RFEA. This is consistent with what we have seen earlier in Figures 6 and 4. The RFEA does still measure a beam, although weak, at $z=80$, while the beam is hardly visible above the noise level in the LIF scan from $z=79 \mathrm{~cm}$. The RFEA thus appears to be more sensitive to beams than LIF. The beam energy measured with the RFEA is consistent with that of the LIF measurements.

Figure 8 shows the beam and background densities (a) and flux (b) derived from the LIF measurements in Figure 7. The densities are integrated over the LIF-measurements as $\int_{a}^{b} f(v) \mathrm{d} v$ and the flux are integrations of $\int_{a}^{b} v f(v) \mathrm{d} v$, where the LIF measurements are used as $f(v)$. Beam densities are taken as the distribution from $v=7.5$ to $14 \mathrm{~km} / \mathrm{s}$, while the backgrounds are taken as everything below $6 \mathrm{~km} / \mathrm{s}$. The flux is calculated in order to compare it to measurements from the RFEA, which measures fluxes. The similarities in the behavior of beam density and beam flux indicate that RFEAmeasurements of beam flux can be regarded as proportional to beam density. Both the beam density and the beam flux seem apparently to decrease like an exponential function with distance from the source for the first three positions $(z=36-56 \mathrm{~cm})$. Between $z=56 \mathrm{~cm}$ and $79 \mathrm{~cm}$, the beam obeys a different exponential decay. The change of exponential decay is seen as a sharp "knee" in the beam density and flux in Figure 8. Exponential fall off has earlier been seen by Keesee et al. ${ }^{1}$ and Cohen et al. ${ }^{14}$ in LIF measurements of beams.

By fitting an exponential function to the data, we find the 1/e folding distance ${ }^{8}$ for both densities and flux to be $14 \mathrm{~cm}$ for the three first measurements and $70-74 \mathrm{~cm}$ for the last three measurements. These 1/e folding distances can be
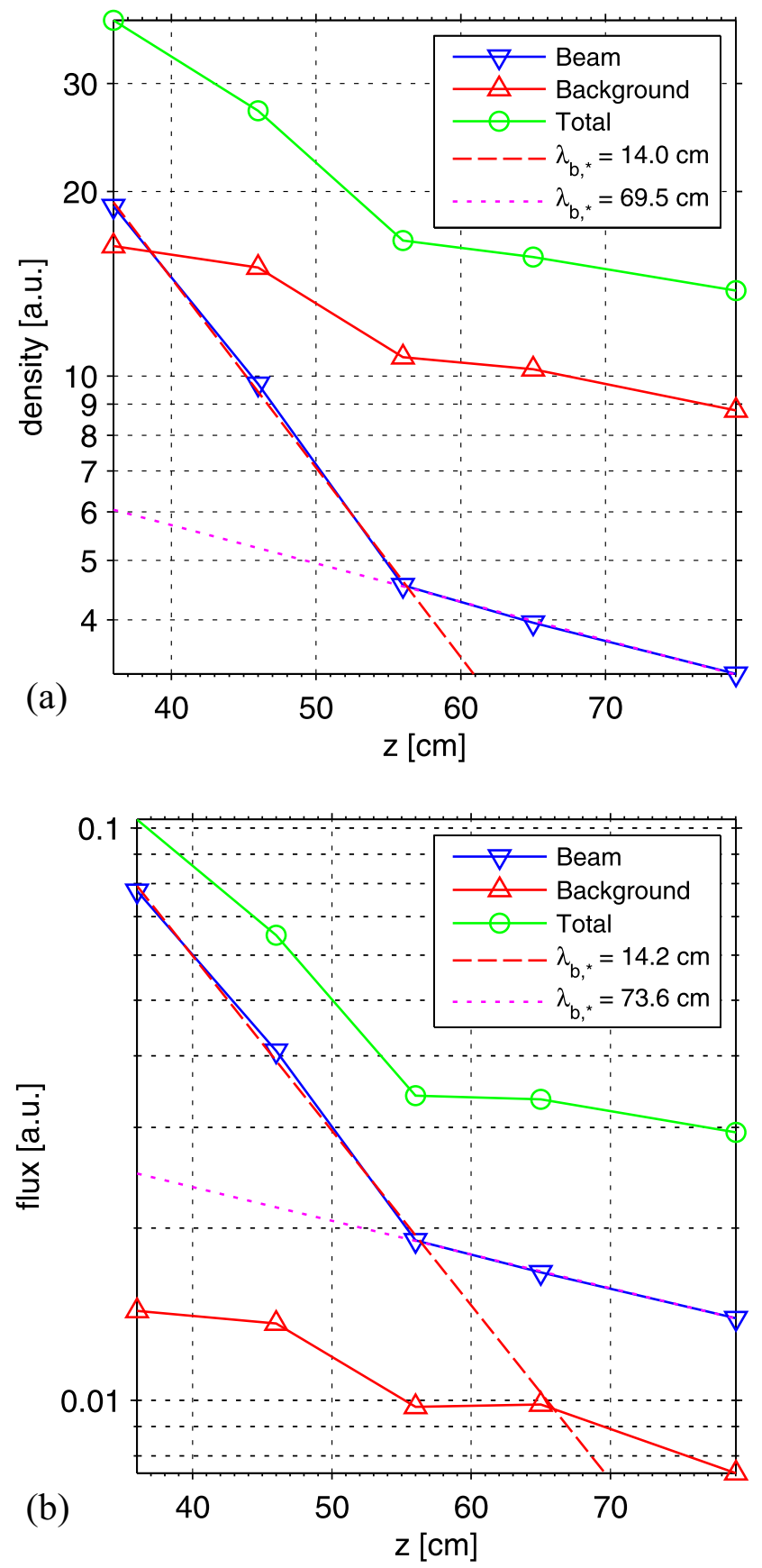

FIG. 8. Integrated beam and background densities (a) and flux (b) calculated from the LIF measurements in Figure 7(a).

interpreted as an effective mean-free-paths for the metastable beam ions, $\lambda_{b, *}$.

\section{Radial extent}

Figure 9 shows a radial profile of flux from RFEA measurements at $z=80 \mathrm{~cm}$ as a function of radial position, from the center of the chamber $(r=0)$ to $r=50 \mathrm{~cm}$. The background distribution stays constant around a plasma potential of $13 \mathrm{~V}$. There is a significant peak in the background density at $r=38 \mathrm{~cm}$, and there is also an increased density at around $20 \mathrm{~cm}$. The beam can be seen at a potential around $30-35 \mathrm{~V}$ being constant in flux from the center and out to about 


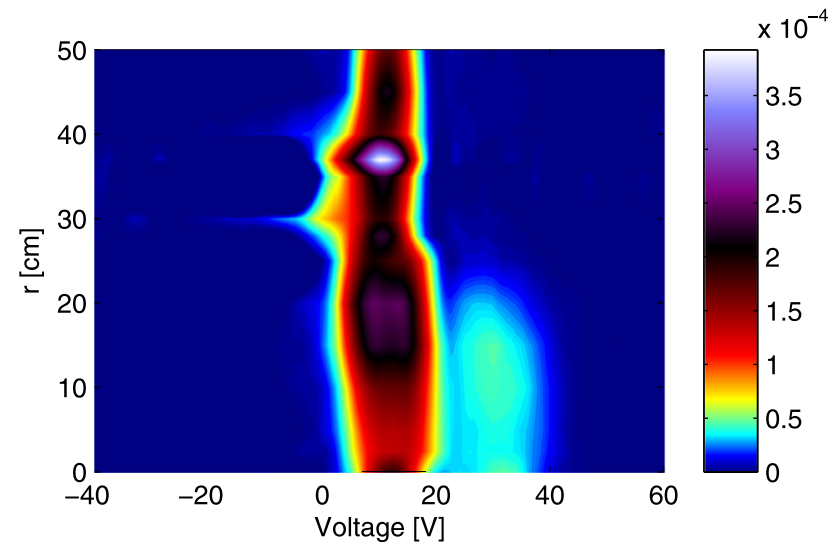

FIG. 9. Radial profiles of RFEA IDF measurements at $z=80 \mathrm{~cm}$ obtained under the same condition as in Figure 7.

$r=20 \mathrm{~cm}$, where the flux decreases gradually until it completely disappears at $r=30 \mathrm{~cm}$.

In Figure 10, the integrated total beam flux from the radial RFEA-scan for two different aperture configurations of the probe are shown. The beam flux is stable out to about $10-15 \mathrm{~cm}$, then it decreases uniformly until $r \approx 30 \mathrm{~cm}$, where it disappears completely.

We can define the beam radius as the radius where the beam density is reduced to half of the beam density in the center. ${ }^{40}$ In the case of Figure 10, the beam radius would be $r_{1 / 2}=20 \mathrm{~cm}$. This is larger than both the source $(r=5 \mathrm{~cm})$ and the chamber of HELIX $(r=7.5 \mathrm{~cm})$. Which indicates that there is some expansion of the beam between the junction $(z=0)$ and the RFEA at $z=80 \mathrm{~cm}$. However, this expansion is much less than the expansion of the magnetic field between HELIX and the LEIA chamber. We can calculate the position of the field line using the expression ${ }^{40}$

$$
\frac{B}{B_{0}}=\left(\frac{r_{0}}{r}\right)^{2}
$$

where $r_{0}$ is the radius of the source and $B_{0}$ is the magnetic field at the source exit.

The footpoint of a field line emerging from $r_{0}=5 \mathrm{~cm}$ in the HELIX source would appear at $r=32 \mathrm{~cm}$ at $z=80 \mathrm{~cm}$. This is close to the small maximum in the background density at $r=37 \mathrm{~cm}$ in Figure 9.

If the beam is expanding along the field line, it will reach $r=20 \mathrm{~cm}$ at $z=51 \mathrm{~cm}$, where the magnetic field is around $60 \mathrm{G}$ and the Larmor radius is $13 \mathrm{~cm}$. This could indicate that the beam detaches from the magnetic field line somewhere around $z=51 \mathrm{~cm}$ and continues as a spatial collimated ion beam. ${ }^{40}$

\section{Pressure dependence}

Figure 11(a) shows LIF measurements at $z=36 \mathrm{~cm}$ at four different gas flows to the source. The gas flows correspond to different pressures in the source, as given in Table II. We observe that the beam velocity decreases with increasing flow/pressure. It is also interesting to note that the beam density at $2.0 \mathrm{sccm}$ flow is significantly higher than at the other pressures while the background densities do not change.

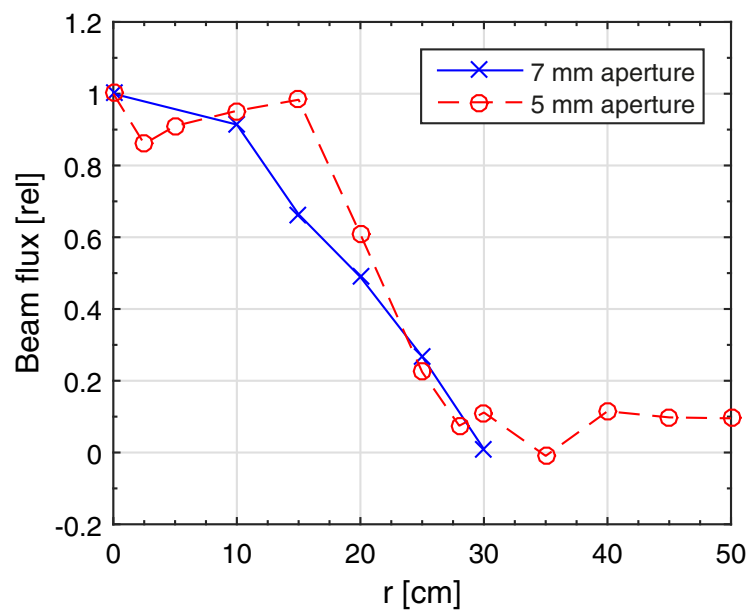

FIG. 10. Measured beam flux from the RFEA probe normalized to the beam flux in the center. Two different probe configurations were used. One with a $7 \mathrm{~mm}$ aperture and one with a $5 \mathrm{~mm}$ aperture.

In Figure 11(b), the LIF measurements are converted to IDF as explained earlier (Figure 6) assuming that beam velocity is unchanged along the z-axis. The plasma potential was taken from the RFEA measurements at $z=80 \mathrm{~cm}$.

Figure 11(c) shows RFEA measurements for the same parameters as in (a) measured at $z=80 \mathrm{~cm}$. The beam
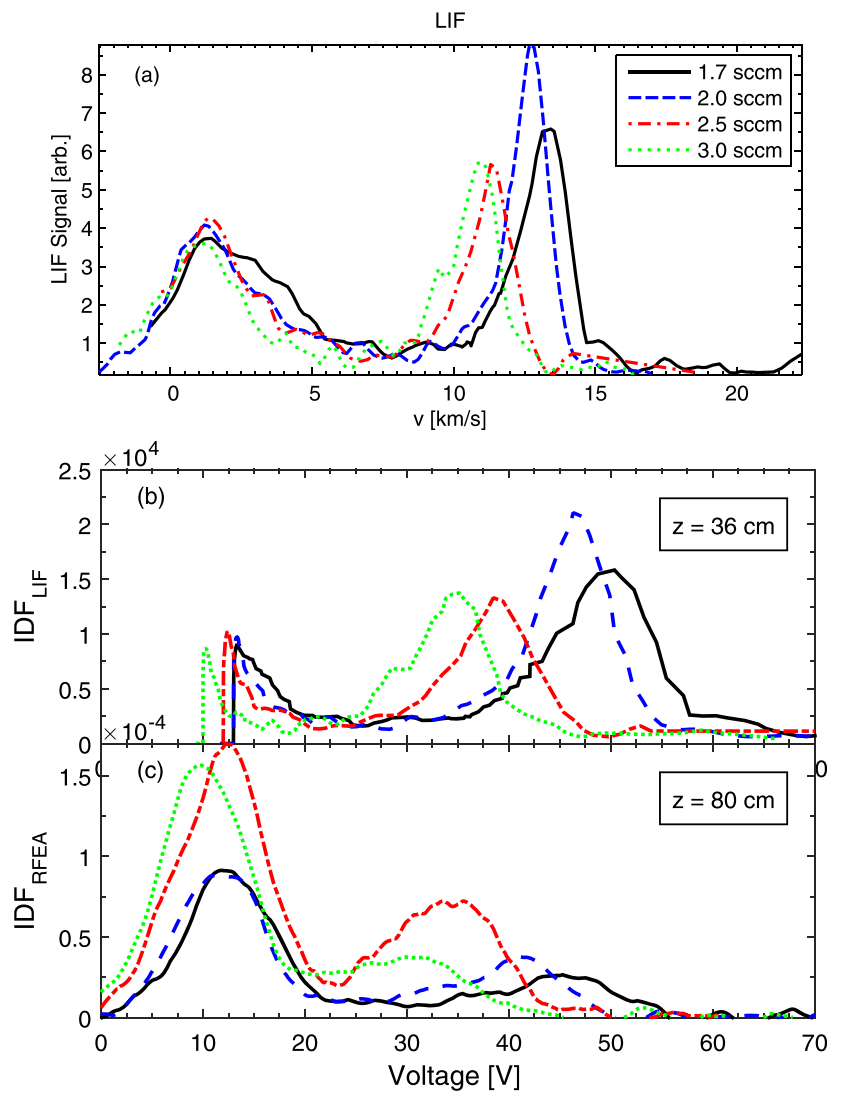

FIG. 11. (a) LIF measurements of velocity distribution function, $f(v)$ in the center of LEIA at $z=36 \mathrm{~cm}$ for different source flow and pressure (pressures given in Table II). RF frequency: $9.5 \mathrm{MHz}$. RF power: $650 \mathrm{~W}$. The LIF data have been filtered with a five point running mean. (b) Derivative of the model collector current calculated from the LIF measurements in (a) using the peak of the background distribution from the RFEA measurements as an estimate of the plasma potential. (c) Derivative of RFEA collector current taken at $z=80 \mathrm{~cm}$ in the center of the chamber. A $5 \mathrm{~mm}$ front aperture was used. 
TABLE II. Pressures, plasma potential, and beam parameters for different flows at $9.5 \mathrm{MHz}$, and $900 \mathrm{G}$ in source for HELIX-LEIA at WVU.

\begin{tabular}{lccccccc}
\hline \hline $\begin{array}{l}\text { Flow } \\
(\mathrm{sccm})\end{array}$ & $\begin{array}{c}P_{\text {HELIX }} \\
(\text { Torr })\end{array}$ & $\begin{array}{c}P_{\text {LEIA }} \\
(\text { Torr })\end{array}$ & $V_{p}(\mathrm{~V})$ & $V_{b}(\mathrm{~V})$ & $E_{b}(\mathrm{eV})$ & $\begin{array}{c}v_{\text {LIF }} \\
(\mathrm{km} / \mathrm{s})\end{array}$ & $\begin{array}{c}E_{b, \mathrm{LIF}} \\
(\mathrm{eV})\end{array}$ \\
\hline 1.7 & $1.5 \times 10^{-4}$ & $5.8 \times 10^{-5}$ & 13 & 47 & 34 & 13.4 & 37 \\
2.0 & $2.8 \times 10^{-4}$ & $6.5 \times 10^{-5}$ & 13 & 43 & 30 & 12.7 & 33 \\
2.5 & $5.3 \times 10^{-4}$ & $7.9 \times 10^{-5}$ & 12 & 35 & 23 & 11.3 & 26 \\
3.0 & $9.2 \times 10^{-4}$ & $8.9 \times 10^{-5}$ & 10 & 32 & 22 & 11.0 & 25 \\
\hline \hline
\end{tabular}

potentials correspond well with the transformed LIFmeasurements, and we see a similar decrease in beam energy with increasing pressure as with LIF. While the beam flux had a maximum at $2.0 \mathrm{sccm}$ in the LIF measurements, beam flux for the RFEA signals is highest at a flow $2.5 \mathrm{sccm} .2 .5$ sccm also have the highest background densities in both measurements. The measurements of the background density at $2.5 \mathrm{sccm}$ and $3.0 \mathrm{sccm}$ are about a factor two higher than those at $1.7 \mathrm{sccm}$ and $2.0 \mathrm{sccm}$. We do not see this effect in the LIF measurements. Thus, we believe that this might be due to a change inside the probe, either in grid transparency or in the resistivity in the probe circuit.

The corresponding HELIX and LEIA pressures for the different flows are given in Table II, together with plasma potential, beam potential, and beam energies from the RFEA measurements in Figure 11(c), and beam velocities and beam energies from the LIF measurements in Figure 11(a).

Figure 12 shows the derived beam energies from LIF and RFEA from Table II plotted as a function of source pressure. We see that beam energies decrease with pressure as expected. ${ }^{1,8}$ The beam energy from the LIF measurements is slightly higher than the ones from RFEA for all pressures. The beam intensity is much lower at $z=80 \mathrm{~cm}$ and the probe would smooth out the peak.

\section{B. UiT Njord}

\section{Axial development}

Figure 13 shows the derivative of the collector current obtained by the axial-RFEA at different positions along the central $z$-axis from $z=36$ to $z=56 \mathrm{~cm}$. We see that the IDF is changing from a single wide distribution close to the source toward a double peaked distribution; a beam at higher energies and a background distribution at lower energies. The single wide distribution is one coming out of the source. It does not seem completely Maxwellian but probably consist of a superposition of several distributions. From emissive probe measurements, we have seen that the plasma potential varies a lot in the source. The plasma would be created at different potentials and then accelerated.

In Figure 13(a), we see that the peak of the distribution is slightly moving toward higher voltages as we go downstream in the plasma chamber. But as noted earlier by Wiebold et al. ${ }^{41}$ and Byhring et al., ${ }^{4}$ the distribution of the beam always stays "within" the initial distribution. The apparent increase in beam energy might be caused by more effective loss of ions at lower energies, due to collisions. In

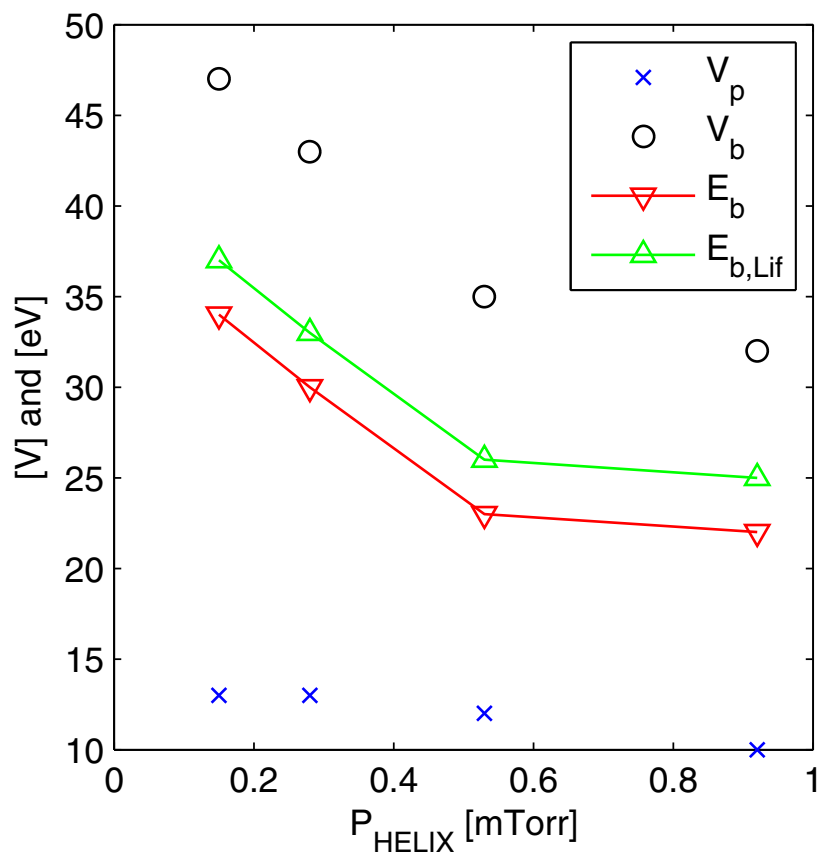

FIG. 12. Beam energy from LIF and RFEA plotted as a function of pressure in the source. The LIF measurements are obtained at $\mathrm{z}=36 \mathrm{~cm}$ and the RFEA measurements at $\mathrm{z}=80 \mathrm{~cm}$.

Figure 13(b), we see that the background distribution grows as the density of the beam decreases.

In Figure 14, the total ion saturation current along with the beam and the background part of the ion collector current are plotted. The currents are proportional to the ion flux to the collector of the probe.

The border between beam and background was found using the same algorithm as used in Figure 4. From around $z \approx 43 \mathrm{~cm}$, this method identifies a beam separated from a background distribution. From this point on, the beam flux decreases nearly exponentially with distance from the source. An exponential fit to the beam flux between $z=43 \mathrm{~cm}$ and $z=52$ gives a $1 / e$-folding distance of $6 \mathrm{~cm}$, which can be seen as an effective ion beam mean free path, $\lambda_{b}$. In contrast, the total flux or ion saturation current, $I_{\text {sat }}$, does not follow an exponential function. Instead, it starts to flatten and gradually stabilize at a certain flux. This implies that the background distribution grows to substitute the beam in the total flux to the probe. The background is found to grow exponentially with a growth constant of $\lambda_{g}=23 \mathrm{~cm}$.

\section{Radial extent}

Figure 15 shows a radial profile of RFEA measurements at $z=55 \mathrm{~cm}$ from the center $(r=0 \mathrm{~cm})$ to $r=18 \mathrm{~cm}$. The background distribution lies between 40 and $60 \mathrm{~V}$ and the beam between 70 and $80 \mathrm{~V}$. The beam flux decreases with radius and disappears after $r=13 \mathrm{~cm}$. The background distribution has a maximum in the center $(r=0)$ and another peak at $r=15 \mathrm{~cm}$.

A radial profile of the total beam flux in units of collector current is shown in Figure 16. The flux is peaked in the center $(r=0)$ and falls of almost linearly with radius out to around $15 \mathrm{~cm}$. The effective beam radius would be 


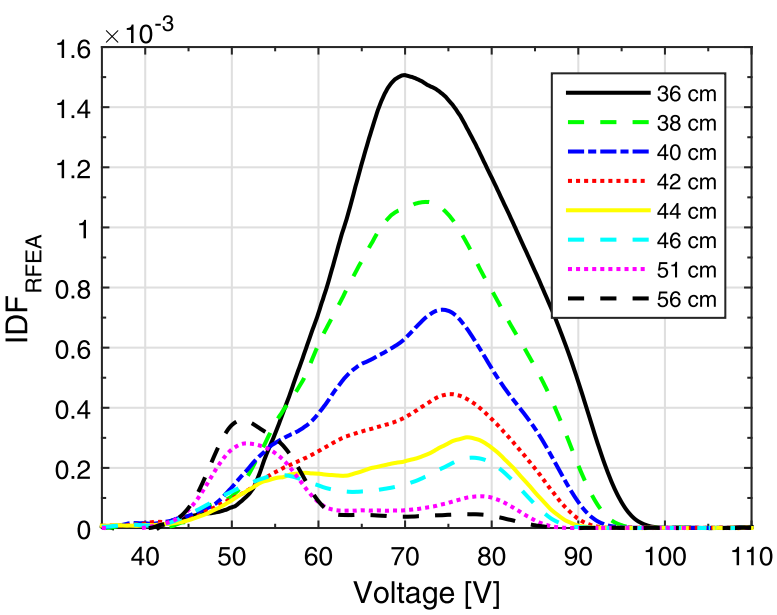

(a)

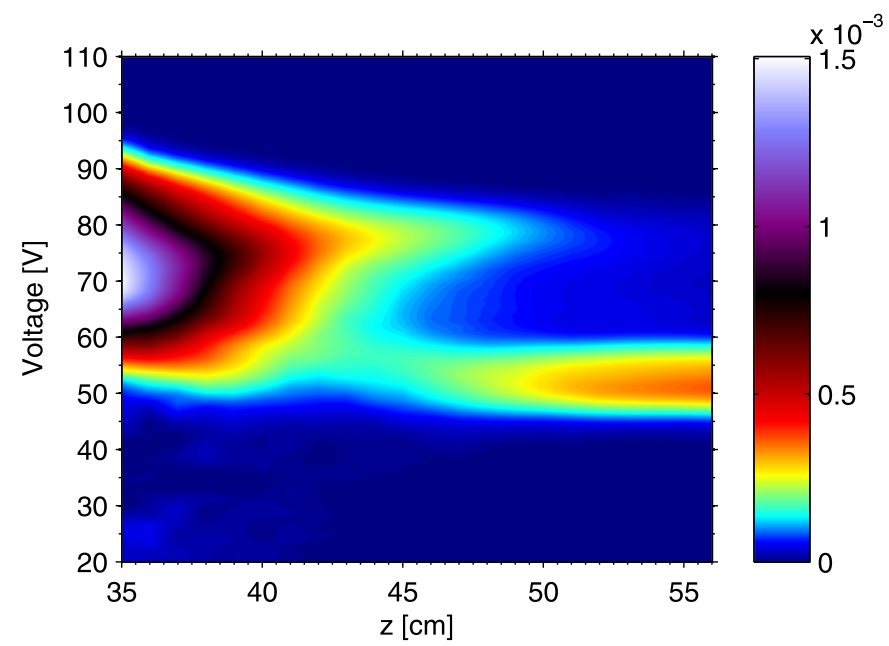

(b)

FIG. 13. RFEA measurements along the central z-axis of Njord. (a) IDFs at eight different positions. (b) A contour plot of IDFs for every $\mathrm{cm}$ from $\mathrm{z}=36$ to $\mathrm{z}=56 \mathrm{~cm}$. The colors represent the signal on the $\mathrm{y}$-axis on (a). Discriminator voltage is displayed along the vertical axis. Both are obtained with a flow of 1.5 sccm resulting in a pressure of $2.8 \times 10^{-4}$ Torr in the expansion chamber.

$r_{1 / 2}=7 \mathrm{~cm}$, which is about the same as the source radius and slightly less than the $10 \mathrm{~cm}$ of the port. But, since the beam flux decreases linearly with radius, a significant part of the beam is actually outside $r=7 \mathrm{~cm}$ and stretches all the way to $r=15 \mathrm{~cm}$. The footpoint of a field line emerging from $r=6.5 \mathrm{~cm}$ in the source would appear at $r=17 \mathrm{~cm}$ at $z=55 \mathrm{~cm}$. This is close to the small maximum at $r=15 \mathrm{~cm}$ in Figure 15.

\section{Pressure dependence}

Figure 17(a) shows LIF measurements at $z=50 \mathrm{~cm}$ for three different gas flows in Njord. The measurements are not

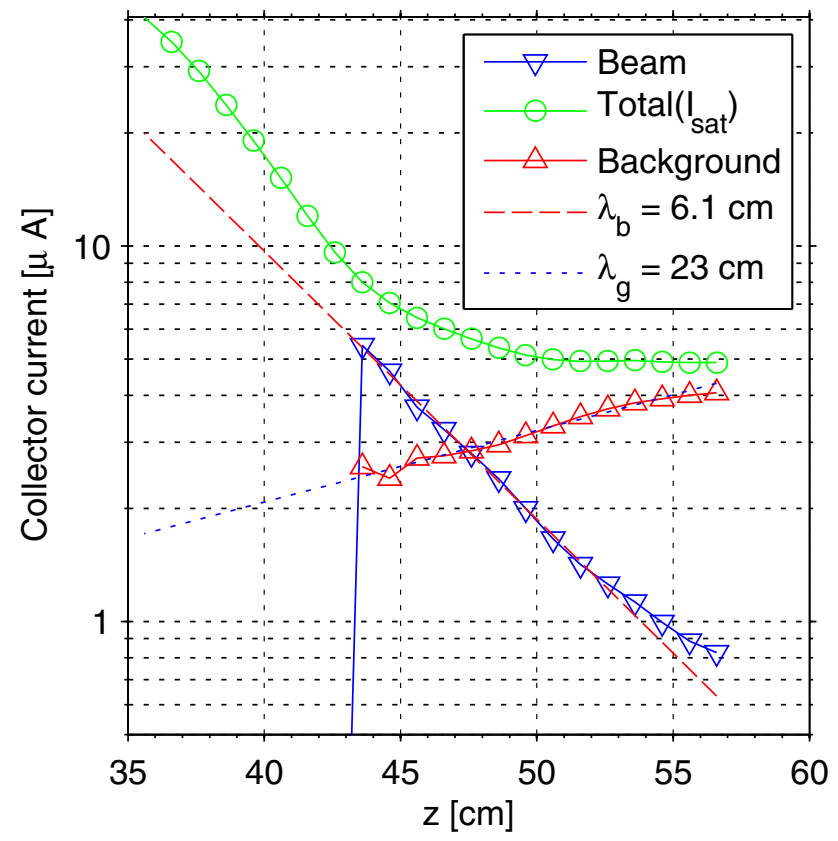

FIG. 14. Logarithmic plot of total ion flux $\left(I_{\text {sat }}\right)$ and beam flux to a RFEAprobe. The red dashed line represents a exponential fit to beam flux in the interval $43 \mathrm{~cm}$ to $52 \mathrm{~cm}$. filtered and are somewhat noisy, but we still can observe a decrease in beam velocity with increasing pressure. Figure 17(b) shows the LIF measurements converted to model RFEA-data using the plasma potential from the RFEA measurements. In Figure 17(c), RFEA-measurements for the same parameters as in Figure 17(a) are shown. In Table III, the pressures for the different flows in Figure 17 are given, as well as plasma potential, beam potential, and beam energy from the RFEA measurements as well as beam velocity and beam energy from the LIF measurements. Figure 18 shows the derived beam energies from LIF and RFEA from Table III plotted versus pressure in Njord, indicating a nearly linear decrease in beam energies with increasing pressure. At all pressures, the beam energy from the LIF measurements are slightly lower than from the RFEA measurements. The measurements are here performed at the same position but at different times. It is possible that the presence of the probe alters the local plasma potential around the probe. A reduced plasma potential would lead to a slightly overestimation of the beam energy. An earlier study by simulations ${ }^{42}$ has shown that the plasma potential measured with an RFEA is slightly lower than the real plasma potential.

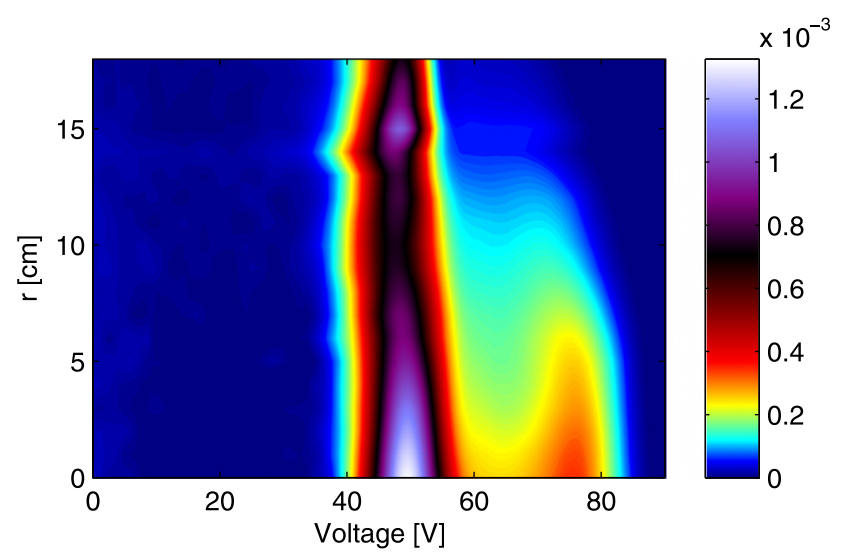

FIG. 15. RFEA measurements in Njord at $1.5 \mathrm{sccm}$. 


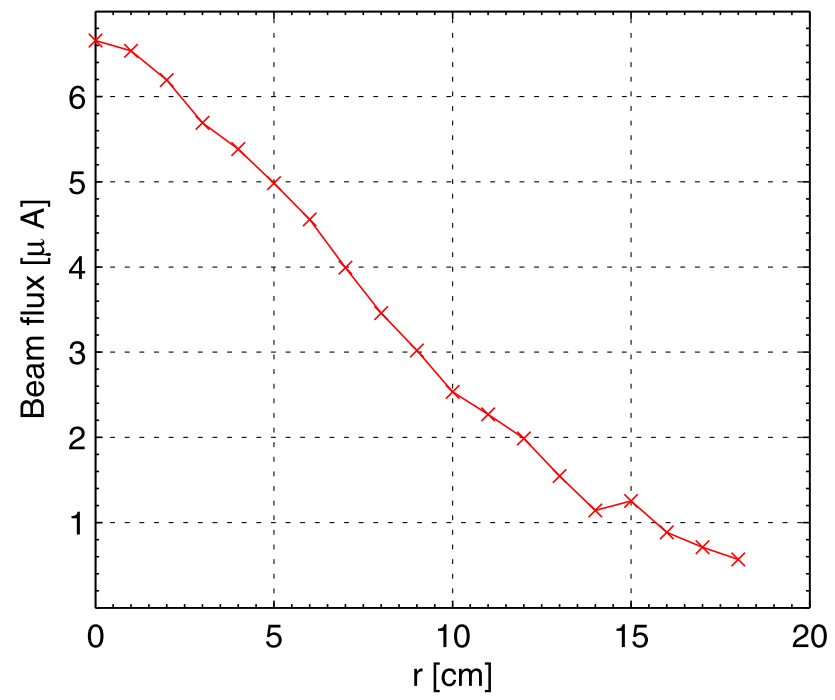

FIG. 16. Radial profile of the beam flux in units of collector current from the RFEA at $z=55 \mathrm{~cm}$ in Njord.

Using axial RFEA-measurements from different pressures, we have done the same analysis as in Figure 14 and derived effective beam ion mean-free path and characteristic growth length for the background. These results are given in Table IV. The cross sections were calculated as $\sigma=k_{b} T / P \lambda$, where $P$ is the measured pressure and the temperature was taken as $297 \mathrm{~K}$. At $2.0 \mathrm{sccm}$, the growth could no longer be
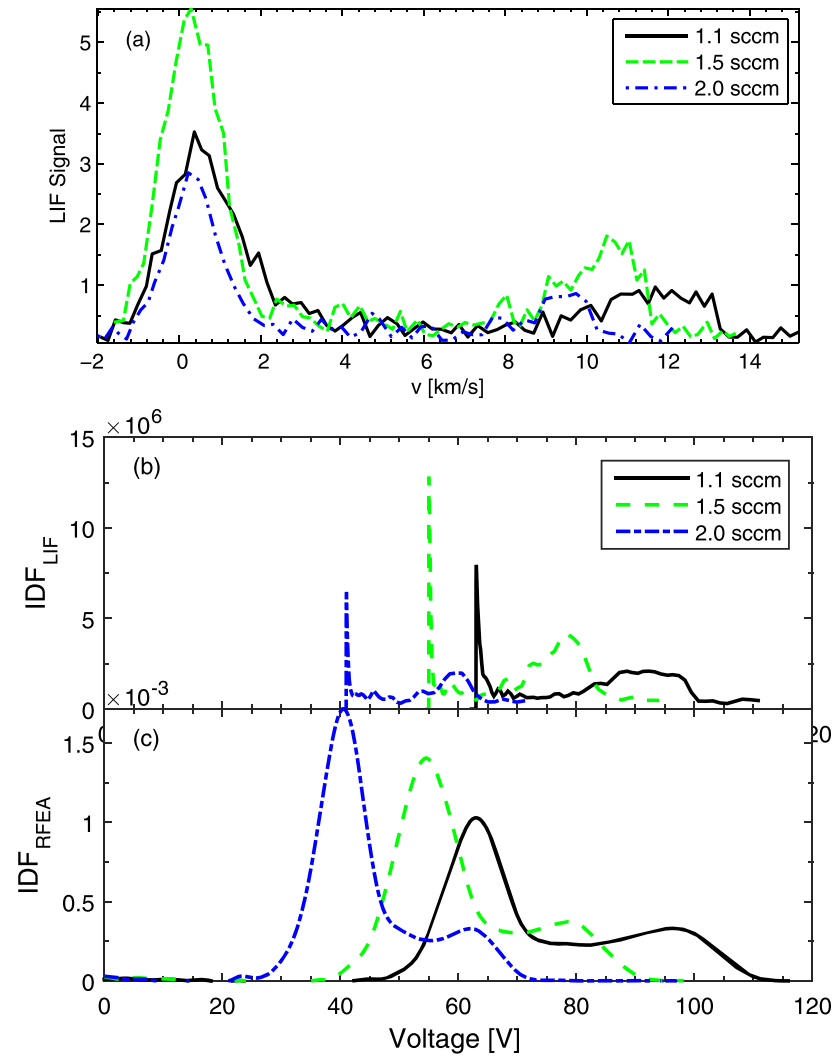

FIG. 17. (a) LIF measurements of velocity distribution function, $f(v)$, in the center of Njord at $z=55 \mathrm{~cm}$. (b) Derivative of the model collector current calculated from the LIF measurements in (a) using the plasma potential from the RFEA measurements. (c) Derivative of RFEA collector current taken at $z=55 \mathrm{~cm}$ in the center of Njord. Pressures are given in Table III.
TABLE III. Pressure, plasma potential, and beam parameters from LIF and RFEA for different flows in Njord.

Flow (sccm) Pressure (Torr) $V_{p}(\mathrm{~V}) V_{b}(\mathrm{~V}) E_{b}(\mathrm{eV}) v_{\mathrm{LIF}}(\mathrm{km} / \mathrm{s}) E_{b, \mathrm{LIF}}(\mathrm{eV})$

\begin{tabular}{lllllll}
\hline 1.1 & $2.1 \times 10^{-4}$ & 63 & 98 & 35 & 12.5 & 32 \\
1.5 & $2.8 \times 10^{-4}$ & 50 & 78 & 28 & 10.7 & 24 \\
2.0 & $3.4 \times 10^{-4}$ & 41 & 63 & 22 & 9.5 & 19 \\
\hline \hline
\end{tabular}

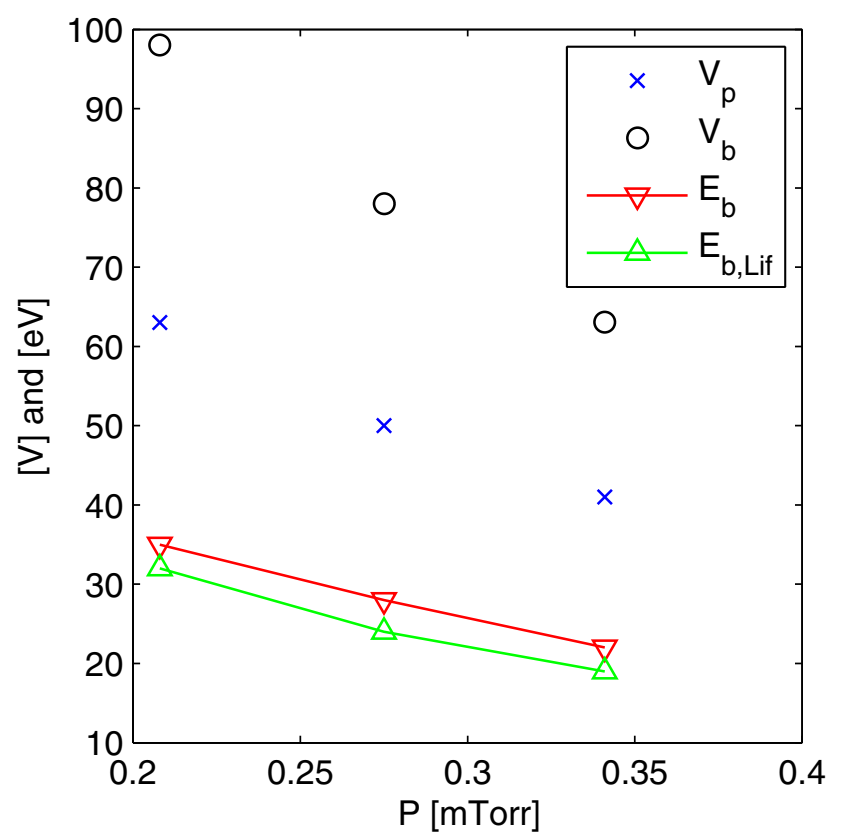

FIG. 18. Beam energy from LIF and RFEA plotted as a function of pressure in Njord including plasma potential, $V_{p}$, and beam potential, $V_{b}$, from the RFEA.

fitted to an exponential function, so no growth cross sections were derived for this pressure.

\section{DISCUSSION}

The method of converting LIF velocity distributions to equivalents of RFEA-IDF provides a direct way of comparing LIF with RFEA-measurements without making too many assumptions about the velocity distribution and the plasma sheath. The only assumptions used are that the LIFmeasurements give a realistic representation of the ion velocity distribution and that the RFEA can provide a realistic plasma potential. Comparing Figure 4 and Fig. 6 shows a good correspondence of the beam measurements, while the background measurements are less accurate with RFEA since the distribution is broadened significantly.

From Figure 7, we see that the RFEA is more sensitive to beam ions than the LIF-technique. The same result was

TABLE IV. Characteristic lengths and effective cross sections from axial RFEA-measurements in Njord.

Flow (sccm) Pressure (Torr) $\lambda_{b}(\mathrm{~cm}) \lambda_{g}(\mathrm{~cm}) \sigma_{b}\left(10^{-20} \mathrm{~m}^{2}\right) \sigma_{g}\left(10^{-20} \mathrm{~m}^{2}\right)$

\begin{tabular}{llllll}
\hline 1.1 & $2.1 \times 10^{-4}$ & 8.4 & 28 & 168 & 51 \\
1.5 & $2.8 \times 10^{-4}$ & 6.1 & 23 & 177 & 47 \\
2.0 & $3.4 \times 10^{-4}$ & 5.2 & $\cdots$ & 168 & $\cdots$ \\
\hline
\end{tabular}


reported by Harvey et al. ${ }^{15}$ The RFEA measures all the ions, not only metastables and it measures fluxes so the signal increase with increasing ion velocity.

At $80 \mathrm{~cm}$ downstream, the LIF-diagnostic hardly see any beam at all, while the RFEA can still detect it. It might be that the beam ions are no longer in the metastable state, ${ }^{1,14}$ but it could just as well be the RFEA is more sensitive to high velocities.

The distribution in the RFEA measurements is much wider than the distributions measured with LIF. There are several possible mechanisms that can create this: RFoscillations in the sheath, ${ }^{39}$ ion focusing inside the probe, ${ }^{36,37}$ and weak ion-ion collisions in the sheath. ${ }^{38}$

The similarities in the behavior of beam density and beam flux seen in Figure 8 indicate that RFEA-measurements of beam flux can be regarded as proportional to the beam density.

The exponential decrease of beam density in LIFmeasurements has earlier been reported by Cohen et al. ${ }^{14}$ and Keesee et al. ${ }^{1}$ who attribute it to collisional quenching of metastables. This quenching would include charge exchange collisions and other loss mechanisms that affect all ions in addition to quenching specific to metastables. ${ }^{17}$

Time of flight for beam ions from the source in LEIA is about $80 \mu$ s and a little bit less for Njord, while the lifetime of the Ar-II $3 d^{2} G_{9 / 2}$ metastable state is about $2 \mathrm{~s},{ }^{43}$ so radiative loss is not contributing significantly to the loss of beam ions.

Looking at the radial measurements in both experiments (Figures 9 and 15), we see that the behavior of the beam and the background plasma is quite similar.

The background density has in both experiments a peak around the footpoint of the magnetic field line from the edge of the source. This is probably due to high energy electrons from the source edge that follows the magnetic field lines. ${ }^{44}$

We observe a discrepancy in the radial beam flux profile between LEIA and Njord. In LEIA (Figure 10) for which the beam flux is flat or almost hollow in the center. This resembles the bimodal profile found by Takahashi et al. ${ }^{40}$ and Cox et $a .^{2}$ On the other hand, the beam profile from Njord (Figure 16) shows a single central peak in beam flux. Cox et al. ${ }^{2}$ observe that the beam profile changes from a bimodal to a single peak with distance from the source. The difference could be due to the different pressures in the expansion chambers. HELIX-LEIA also have a stronger magnetic field gradient that could lead to a stronger expansion in the source. The beam in LEIA is found to be wider than the source, while in Njord, the beam stays at approximate the same width as the source. Takahashi et al. ${ }^{40}$ found in a similar device that the beam only expands close to the source. At some point, the ions detach from the magnetic field and continue as a spatially collimated ion beam, although Cox et al. ${ }^{2}$ have found a small beam divergence. Takahashi and Fujiwara ${ }^{45}$ only find a significant beam divergence for higher pressures and suggest that both a convex double layer and a radial electric field contribute to the divergence.

Observing the measurements of beam width in LEIA (Figure 10), it is plausible that the beam ions stays frozen to the magnetic field line until around $z=51 \mathrm{~cm}$, where they detach and continue as a spatially collimated ion beam. This can explain the sharp "knee" and the two different mean-free-paths found in Figure 8. The first two positions, at $z=36 \mathrm{~cm}$ and $z=46 \mathrm{~cm}$, are in the region where the beam is expanded by the magnetic field. This gives rise to the artificially low mean-freepath of $14 \mathrm{~cm}$. The last three positions $(z=56-79 \mathrm{~cm})$ are obtained in the region of the collimated ion beam and should give a more correct measurement of the mean-free-path.

The ion-neutral collision cross section should be about $60 \times 10^{-20} \mathrm{~m}^{2}$ for beam energies around $25 \mathrm{eV},{ }^{24,46}$ which would correspond to a mean-free-path of $\lambda_{b}=53 \mathrm{~cm}$. This is slightly shorter than the $70 \mathrm{~cm}$ we measure for the three last measurements in Figure 8. A quenching mean-free-path of $70 \mathrm{~cm}$ would correspond to a quenching cross section of $40 \times 10^{-20} \mathrm{~m}^{2}$. This discrepancy could be due to contributions from the noise to the integrated beam after $z=65 \mathrm{~cm}$, where the beam intensity is approaching the noise level.

In Njord, we do not observe beam expansion. At $z=55 \mathrm{~cm}$, the beam is about the same width as the source diameter, although some diffusion of the beam is observed (Figure 16).

In Figure 13, we see a similar behavior in the axial RFEA-measurements as we see in the LIF-measurements in LEIA. The RFEA-measurements exhibit the same type of exponential decrease of the beam flux (Figure 14). Exponential decrease dominates the total flux in the region before the beam is detected. In addition, the background grows exponentially with distance from the source. This increase in background density with $z$ indicates that the background plasma is not coming from the source but created locally by ionization of neutrals, through charge exchange collisions. This type of growth in background densities is not seen in LEIA, instead the density decreases with $z$ (Figure 8). This could be explained by the large distance from the source in LEIA. In Njord, the beam is stronger since it is closer to the source. In addition, Njord also has a higher neutral density that leads to a shorter mean-free-path and more chargeexchange collisions producing background ions.

The derived effective beam collisional cross section from data at three different pressures in Table IV is surprisingly consistent around $170 \times 10^{-20} \mathrm{~m}^{2}$. This result is higher than the expected ion-neutral collision cross section of $60 \times 10^{-20} \mathrm{~m}^{2} \cdot{ }^{24,46}$ We cannot completely rule out that magnetic expanding contribute to this result. We have not seen any other indication of magnetic expansion in the beam measurements in Njord. It is also possible that radial electric fields or a convex double-layer-structure contributes to a small divergence in the beam and affects the measurements of collisional cross sections. ${ }^{45}$

In Figures 12 and 18, we observe that beam energies decrease with pressure. Keesee et al. ${ }^{1}$ suggested that beam energy scales with $1 / P^{2}$. Lieberman and Charles ${ }^{8}$ present a more complex model for double layer strength, which defines beam energy, that has a similar dependence on pressure. Both the beam energies form LEIA and Njord appears to follow a similar trend.

The diode-LIF system at Njord is capable of detecting a beam at all the pressures tested in the same distance to the source as the RFEA. This shows that fairly weak $(20 \mathrm{~mW})$ diode-LIF can be used to measure ion beams given enough filtering/integration time at the lock-in amplifier. 


\section{CONCLUSION}

We have developed a new method for converting LIFvelocity distributions to equivalents of RFEA-IDF, which provides a direct way of comparing LIF with RFEAmeasurements. The method shows good agreement between measurements in two plasma experiments (HELIX-LEIA at WVU and Njord at UiT).

The RFEA seems to be more sensitive to beam ions, and is able to measure an ion beam with densities too low for the LIF to resolve.

In Njord, the beam is found to have about the same width as the diameter of the source. In LEIA, the beam is slightly wider than the source, which indicates some sort of beam expansion. The change in effective mean-free-path seen in the axial beam measurements in LEIA is consistent with magnetic expansion of the beam for the two first measurements.

A peak in the background density is found in the radial RFEA measurements in both experiments, close to the magnetic field line mapping to the edge of the source. This is most likely a signature of high-energy electrons from the source, ionizing the neutral gas.

Beam densities drop off exponentially with distance from the source, both in LIF and RFEA measurements. We calculate the effective quenching cross section in LEIA to be $\sigma_{b, *}=4 \times 10^{-19} \mathrm{~m}^{2}$, which is lower than expected. The effective beam collisional cross section in Njord was calculated to be $\sigma_{b}=1.7 \times 10^{-18} \mathrm{~m}^{2}$, which is higher than expected.

The increase in background densities with distance from the source seen in Njord indicates that the background plasma is produced locally in the expansion chamber, most likely due to charge exchange collisions with the beam ions.

\section{ACKNOWLEDGMENTS}

Parts of this research were done during an extended visit at West Virgina University with support from University of Troms $\varnothing$. The authors would like to thank staff and students at the plasma lab at West Virgina University and at University of Troms $\varnothing$. Thanks also to the technical staff at both institutions for their skilled and helpful assistance.

${ }^{1}$ A. M. Keesee, E. E. Scime, C. Charles, A. Meige, and R. Boswell, Phys. Plasmas 12, 093502 (2005).

${ }^{2}$ W. Cox, C. Charles, R. W. Boswell, and R. Hawkins, Appl. Phys. Lett. 93, 071505 (2008).

${ }^{3}$ X. Sun, S. A. Cohen, E. E. Scime, and M. Miah, Phys. Plasmas 12, 103509 (2005).

${ }^{4}$ H. S. Byhring, C. Charles, A. Fredriksen, and R. W. Boswell, Phys. Plasmas 15, 102113 (2008)

${ }^{5}$ T. Schröder, O. Grulke, and T. Klinger, EPL Europhys. Lett. 97, 65002 (2012).

${ }^{6}$ C. Biloiu, X. Sun, E. Choueiri, F. Doss, E. Scime, J. Heard, R. Spektor, and D. Ventura, Plasma Sources Sci. Technol. 14, 766 (2005).

${ }^{7}$ K. Takahashi, Y. Shida, T. Fujiwara, and K. Oguni, IEEE Trans. Plasma Sci. 37, 1532 (2009).

${ }^{8}$ M. A. Lieberman and C. Charles, Phys. Rev. Lett. 97, 045003 (2006).

${ }_{9}^{9}$ A. Meige, R. Boswell, C. Charles, J.-P. Boeuf, G. Hagelaar, and M. Turner, IEEE Trans. Plasma Sci. 33, 334 (2005).
${ }^{10}$ A. Fruchtman, Phys. Rev. Lett. 96, 065002 (2006).

${ }^{11}$ N. Singh, Plasma Phys. 24, 639 (1982).

${ }^{12}$ C. Charles and R. Boswell, Appl. Phys. Lett. 82, 1356 (2003).

${ }^{13}$ C. Charles and R. W. Boswell, Phys. Plasmas 11, 1706 (2004).

${ }^{14}$ S. A. Cohen, N. S. Siefert, S. Stange, R. F. Boivin, E. E. Scime, and F. M. Levinton, Phys. Plasmas 10, 2593 (2003).

${ }^{15}$ Z. Harvey, S. C. Thakur, A. Hansen, R. Hardin, W. S. Przybysz, and E. E. Scime, Rev. Sci. Instrum. 79, 10F314 (2008).

${ }^{16}$ G. Severn, D. A. Edrich, and R. McWilliams, Rev. Sci. Instrum. 69, 10 (1998).

${ }^{17}$ M. J. Goeckner, J. Goree, and T. E. Sheridan, Phys. Fluids B: Plasma Phys. (1989-1993) 3, 2913 (1991).

${ }^{18}$ R. F. Boivin and E. E. Scime, Rev. Sci. Instrum. 74, 4352 (2003).

${ }^{19}$ X. Sun, C. Biloiu, R. Hardin, and E. E. Scime, Plasma Sources Sci. Technol. 13, 359 (2004).

${ }^{20}$ S. C. Thakur, A. Hansen, and E. E. Scime, Plasma Sources Sci. Technol. 19, 025008 (2010).

${ }^{21}$ P. A. Keiter, E. E. Scime, and M. M. Balkey, Phys. Plasmas 4, 2741 (1997).

${ }^{22}$ E. E. Scime, P. A. Keiter, M. M. Balkey, R. F. Boivin, J. L. Kline, M. Blackburn, and S. P. Gary, Phys. Plasmas 7, 2157 (2000).

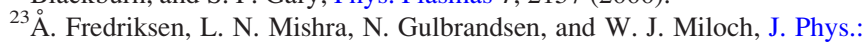
Conf. Ser. 257, 012019 (2010).

${ }^{24} \mathrm{M}$. Lieberman and A. Lichtenberg, Principles of Plasma Discharges and Materials Processing (Wiley, New York, 2005).

${ }^{25}$ D. Gahan, S. Daniels, C. Hayden, D. O. Sullivan, and M. B. Hopkins, Plasma Sources Sci. Technol. 21, 015002 (2012).

${ }^{26}$ C. Charles, R. W. Boswell, and R. K. Porteous, J. Vac. Sci. Technol. A 10, 398 (1992).

${ }^{27}$ E. Leal-Quiros and M. Prelas, IEEE Trans. Plasma Sci. 16, 661 (1988).

${ }^{28}$ C. Böhm and J. Perrin, Rev. Sci. Instrum. 64, 31 (1993).

${ }^{29}$ G. A. Emmert, R. M. Wieland, A. T. Mense, and J. N. Davidson, Phys. Fluids 23, 803 (1980).

${ }^{30}$ W. J. Miloch, N. Gulbrandsen, L. N. Mishra, and A. Fredriksen, Appl. Phys. Lett. 97, 261501 (2010).

${ }^{31}$ N. Gulbrandsen, W. J. Miloch, and A. Fredriksen, Contrib. Plasma Phys. 53, 27 (2013).

${ }^{32}$ D. N. Hill, S. Fornaca, and M. G. Wickham, Rev. Sci. Instrum. 54, 309 (1983).

${ }^{33}$ A. K. Hansen, M. Galante, D. McCarren, S. Sears, and E. E. Scime, Rev. Sci. Instrum. 81, 10D701 (2010).

${ }^{34}$ J. Carr, Jr., "Laser induced fluorescence studies of electrostatic double layers in an expanding helicon plasma," Ph.D. thesis, West Virginia University, 2013.

${ }^{35}$ P. C. Stangeby, "Further aspects of the sheath," in The Plasma Boundary of Magnetic Fusion Devices (Taylor and Francis, 2000), pp. 629-655.

${ }^{36}$ Y. Hamada, Y. Kawasumi, H. Iguchi, A. Fujisawa, Y. Abe, and M. Takahashi, Rev. Sci. Instrum. 65, 1606 (1994).

${ }^{37}$ V. Kanarov, D. Siegfried, P. Sferlazzo, A. Hayes, and R. Yevtukhov, Rev. Sci. Instrum. 79, 093304 (2008).

${ }^{38}$ S. A. Andersen, V. O. Jensen, P. Michelsen, and P. Nielsen, Phys. Fluids 14, 728 (1971).

${ }^{39}$ C. Charles, A. W. Degeling, T. E. Sheridan, J. H. Harris, M. A. Lieberman, and R. W. Boswell, Phys. Plasmas 7, 5232 (2000).

${ }^{40}$ K. Takahashi, Y. Itoh, and T. Fujiwara, J. Phys. D: Appl. Phys. 44, 015204 (2011).

${ }^{41}$ M. Wiebold, Y.-T. Sung, and J. E. Scharer, Phys. Plasmas 18, 063501 (2011).

${ }^{42}$ W. J. Miloch, N. Gulbrandsen, L. N. Mishra, and A. Fredriksen, Phys. Plasmas 18, 083502 (2011).

${ }^{43}$ P. Lundin, J. Gurell, L.-O. Norlin, P. Royen, S. Mannervik, P. Palmeri, P. Quinet, V. Fivet, and E. Biémont, Phys. Rev. Lett. 99, 213001 (2007).

${ }^{44}$ K. Takahashi, C. Charles, R. Boswell, W. Cox, and R. Hatakeyama, Appl. Phys. Lett. 94, 191503 (2009).

${ }^{45}$ K. Takahashi and T. Fujiwara, Appl. Phys. Lett. 94, 061502 (2009).

${ }^{46}$ W. McDaniel, J. Mitchell, and E. Rudd, Atomic Collisions: Heavy Particle Projectiles (Wiley, New York, 1993). 